\title{
Microplastic sampling techniques in freshwaters and sediments: a review
}

\author{
Nastaran Razeghi ${ }^{1} \cdot$ Amir Hossein Hamidian ${ }^{1,2}$ (1) Chenxi Wu $\mathbf{u}^{3,4} \cdot$ Yu Zhang ${ }^{2,4} \cdot$ Min Yang ${ }^{1,2,4}$
}

Received: 18 December 2020/Accepted: 13 March 2021 / Published online: 18 May 2021

(C) The Author(s), under exclusive licence to Springer Nature Switzerland AG 2021

\begin{abstract}
Pollution by microplastics is of increasing concern due to their ubiquitous presence in most biological and environmental media, their potential toxicity and their ability to carry other contaminants. Knowledge on microplastics in freshwaters is still in its infancy. Here we reviewed 150 investigations to identify the common methods and tools for sampling microplastics, waters and sediments in freshwater ecosystems. Manta trawls are the main sampling tool for microplastic separation from surface water, whereas shovel, trowel, spade, scoop and spatula are the most frequently used devices in microplastic studies of sediments. Van Veen grab is common for deep sediment sampling. There is a need to develop optimal methods for reducing identification time and effort and to detect smaller-sized plastic particles.
\end{abstract}

Keywords Microplastic pollution $\cdot$ Freshwater systems $\cdot$ Polymer $\cdot$ Sampling $\cdot$ Water $\cdot$ Sediment

\section{Introduction}

Ecosystems and their different organisms have been widely impacted by anthropogenic activities such as the discharge of pollutants (Hamidian et al. 2019; Mirzajani et al. 2016, 2015; Padash Barmchi et al. 2015; Rezaei Kalvani et al. 2019), including emerging contaminants (Jafari Ozumchelouei et al. 2020) such as microplastics. Endurance, flexibility, lightweight, being low cost and being waterproof allows for plastic use in different applications, leading to their accumulation in the environment (Pellini et al. 2018; Razeghi et al. 2020). Plastic materials are used in a wide variety of

Amir Hossein Hamidian

a.hamidian@ut.ac.ir

1 Department of Environmental Science and Engineering, Faculty of Natural Resources, University of Tehran, P.O. Box 4314, Karaj 31587-77878, Iran

2 State Key Laboratory of Environmental Aquatic Chemistry, Research Center for Eco-Environmental Sciences, Chinese Academy of Sciences, Beijing 100085, China

3 State Key Laboratory of Freshwater Ecology and Biotechnology, Institute of Hydrobiology, Chinese Academy of Sciences, Wuhan 430072, China

4 University of Chinese Academy of Sciences, Beijing 100049, PR China markets and industries, including packaging, building and construction, electrical, agriculture, consumer and household appliances such as toothpaste and facial scrubbers, etc. (Wu et al. 2018). According to data from Plastics Europe, world production of plastics reached 335 million tons in 2016 (Plastics Europe 2018). It is estimated that by 2050, this may increase to 33 billion tons (Horton et al. 2017). By then, 12,000 million metric tons (Mt) of plastic waste will have been accumulated in landfills and natural environments (Geyer et al. 2017). Recently, a sharp increase is induced in plastic waste production such as masks, gloves, and plastic shopping bags by the coronavirus disease (COVID-19) pandemic (Gorrasi et al. 2020). Once plastics are discharged into aquatic environments, they can persist for up to 50 years. Complete plastic mineralization may take hundreds or thousands of years (Holland et al. 2016).

Microplastics are generally defined as plastic particles smaller than a specified upper size limit $(<5 \mathrm{~mm})$. However, sometimes smaller size limits have also been proposed. Currently, there is no specific lower size cutoff for this definition (Connors et al. 2017). Since the 1970s when the first reports of micro-sized particles were published, marine plastic pollution has been of concern (Carpenter and Smith 1972; Carpenter et al. 1972; Colton et al. 1974). Plastic debris in the ocean was recognized by the United Nations Environment Program (UNEP) as an emerging 
global environmental issue (Kershaw et al. 2011). However, "microplastics" were first described by Thompson and colleagues in 2004. They reported the occurrence and presence of plastics around $50 \mu \mathrm{m}$ in size on shorelines and in water column (Thompson et al. 2004). Microplastics are commonly defined as plastic particles with sizes below $5 \mathrm{~mm}$ (Hidalgo-Ruz et al. 2012).

Depending on the way in which microplastics are produced, they can be classified into two classes as primary or secondary. Primary microplastics are small plastic particles released directly into the environment by domestic and industrial effluents, spills and sewage discharge or indirectly by runoff. Secondary microplastics are formed as a result of fragmentation of larger plastic particles already present in the environment. Fragmentation takes place due to UV radiation (photo-oxidation), mechanical transformation (e.g., via waves abrasion) and biological degradation by microorganisms (de Sá et al. 2018; Thompson et al. 2009).

There are hundreds of commercially available plastic materials. Polypropylene and low- and high-density polyethylene are the three most common used plastic polymers in packaging. Polyvinyl chloride, polyurethanes, polyethylene terephthalate, polystyrene and polyester are also widely used due to their various applications (Plastics Europe 2018).

In terms of shape, microplastics fall into five main groups. Fragments are three-dimensional and hard jaggededged particles. Pellets have hard rounded shape. Fibers are fibrous or thin uniform plastic strands, and films are thin, two-dimensional plastic pieces. Foam is a mass of tiny bubbles (i.e., styrofoam-type material) (Anderson et al. 2017; Rezania et al. 2018). Using different measuring instruments, different results may be obtained in terms of shape, size and type of microplastics. The major sampling techniques are shown in Fig. 1.

Despite extensive research on microplastics in marine environments, less effort has been made to monitor microplastics in freshwaters. Freshwaters include water in ice sheets, ice caps, glaciers, icebergs, bogs, ponds, lakes, rivers, streams, marshlands, wetlands and groundwater. Freshwaters are generally characterized as having low concentrations (less than $1000 \mathrm{mg} \mathrm{L}^{-1}$ ) of dissolved salts and other total dissolved solids (American Meteorological Society 2012). Although the ocean floor is considered to be the ultimate fate of marine microplastics, inland water bodies might also be a terminal or transient sink for microplastics (Z. F. Wang et al. 2018b). Freshwater bodies can have comparable plastic concentrations to marine waters.

Microplastics can cause several harmful physical effects on humans and living organisms through such mechanisms as entanglement and ingestion. They can cause blockage of the gastrointestinal tract or inflammatory responses and consequently a range of adverse effects. Some effects include lower energy reserves, reduced reproduction/growth, oxidative damage, metabolism disruption, cellular lesions, starvation and even death (Ding et al. 2018; Ogonowski et al. 2016). Exposure of microplastics to a cohort of human adults (hand-face skin, head hair and saliva) has been reported (Abbasi and Turner 2021).

The trophic transfer of microplastics in the aquatic food web has been demonstrated by researchers (Farrell and Nelson 2013; Setälä et al. 2014). Microplastics' large surface area to volume ratio provides a high association potential for environmental contaminants. Microplastics have an affinity for certain hazardous hydrophobic organic chemicals, non-essential trace elements and persistent organic pollutants. Some examples include polychlorinated biphenyls, dichlorodiphenyltrichloroethane, additives, plasticizers and heavy metals (Brennecke et al. 2016; Hartmann et al. 2017; Holland et al. 2016; Koelmans et al. 2016; Naqash et al. 2020).

Wastewater treatment plants receive large amounts of microplastics among other pollutants. However, efforts of treatment (Mojoudi et al. 2018, 2019) including biological methods (Alavian et al. 2018; Hamidian et al. 2016; Mansouri et al. 2013; Mirzajani et al. 2017) remove most of these emerging pollutants.

Different microplastics treatment methods include sorption and filtration, biological removal and ingestion, and chemical treatments. Sorption of microplastics on green algae is based on charged microplastics. Membrane technology regarding durability, influent flux, size and concentration of the microplastics in water and wastewater have shown good efficiency. Coagulation and agglomeration processes, using Fe-based and Al-based salts, are also reported. Electrocoagulation technique and photocatalytic degradation using $\mathrm{TiO}_{2}$ and $\mathrm{ZnO}$ semiconductors are used as robust and environmentally friendly techniques. Microplastics ingestion by organisms is also discussed as a removal strategy. However, sorption and filtration processes coupled with membrane bioreactors lead to higher microplastics removal compared to other methods (Hamidian et al. 2021; Padervand et al. 2020). Othman and coworkers reviewed microplastics degradation through enzymatic processes (Othman et al. 2021). ZnO nanorod photocatalysts excited by visible light were used to degrade low density polyethylene film in water (Tofa et al. 2019).

Inland waters and marine environments are facing similar issues related to microplastics presence. However, some differences like physical and chemical characteristics of water cannot be ignored. Here we review techniques for sampling microplastics in waters and sediments with focus of the following issues:

a. What is the evolution of the number of scientific studies on microplastics in freshwater and sediment? 
Prevalent microplastic sampling tools in different freshwater compartments (water and sediment)

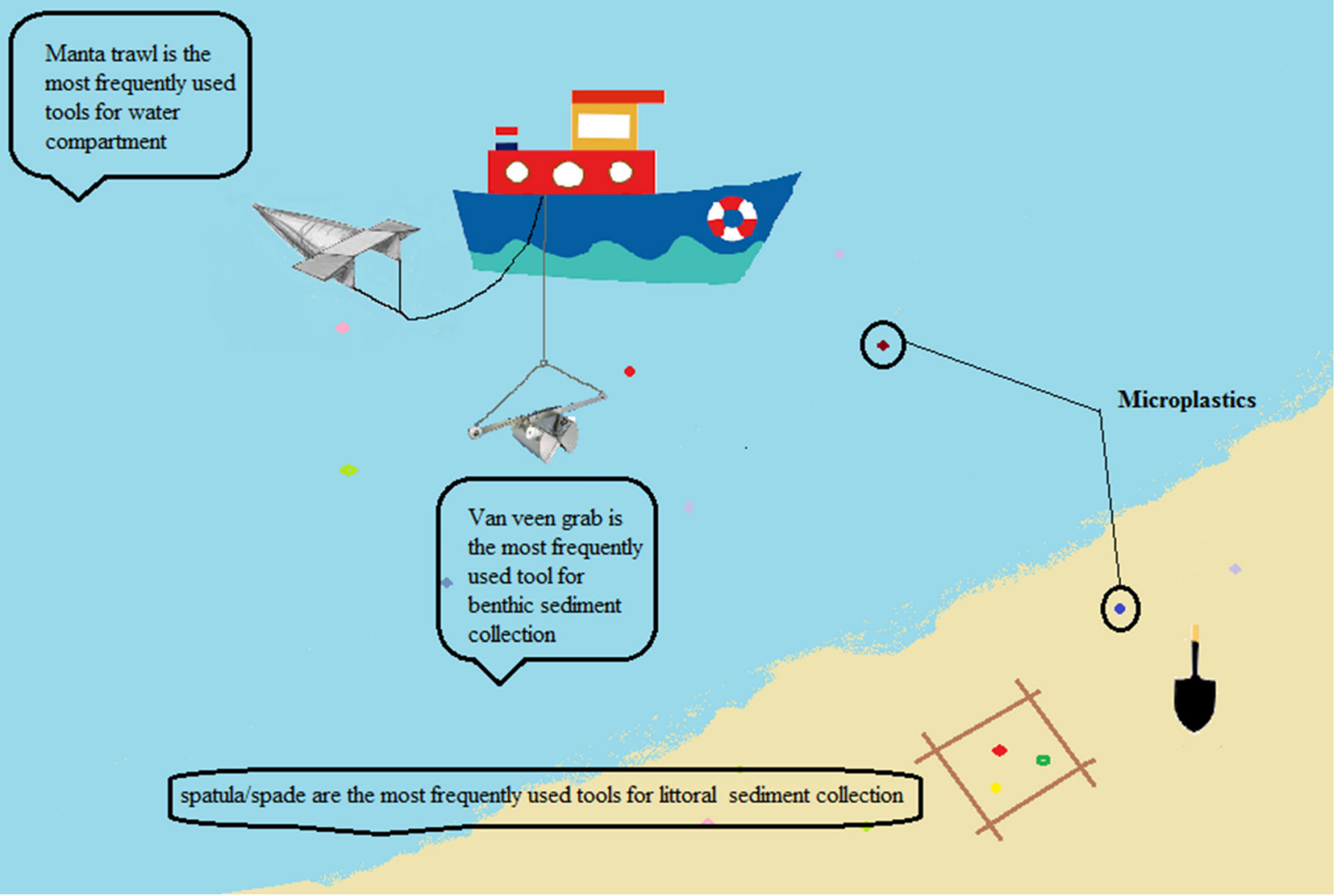

Fig. 1 Common techniques to sample microplastics

b. Which freshwater compartments are more commonly investigated for microplastics?

c. Which sampling matrix, water or sediment, is most frequently studied?

d. What are the most common sampling methods in water and sediment studies?

e. What are the advantages and disadvantages of sampling methods?

\section{Data acquisition}

Literature was gathered through online search in the ISI Website of Knowledge, Science Direct and Google Scholar using keywords and phrases including "microplastic" OR AND "freshwater", OR AND "plastic particle", OR AND "plastic fragment", OR AND "pellets" OR AND "river" OR AND "estuary" OR AND "lake". The retrieved articles were then screened by study area, of which studies in water and sediment of inland water systems were selected including rivers, estuaries, lakes, reservoirs, estuaries, etc.
After identifying candidate research, the abstracts of all studies reporting microplastics in freshwater ecosystems were studied. It is worthy noticing that microplastic research solely on microplastics in freshwater species was excluded. However, a combination of water or sediment studies with biota or all three (water, sediment and biota) were included simultaneously. A total of 150 published pieces of research between 2010 and 2020 were retrieved and evaluated. Details of each study were recorded in an EXCEL spreadsheet for subsequent analysis. This information was used to determine the extent and depth of current microplastic research and to identify important data gaps.

\section{Microplastics presence in freshwater environments}

Research papers with an emphasis on microplastics in inland water bodies are mostly published in the last ten years. Microplastics have been recorded along shorelines of the Tamar estuary, UK (Browne et al. 2010). In two urban rivers 
(the Los Angeles River and the San Gabriel River), Southern California, microplastics were found 16 times more abundant than macroplastics and three times heavier than the bigger particles (Moore et al. 2011). Zbyszewski and Corcoran (2011) scrutinized the distribution and degradation of plastic particles along the beaches of Lake Huron, Canada (Zbyszewski and Corcoran 2011). The first ecosystemic review, assessing microplastics in different compartments, including water, sediment and biota, was reported by Faure et al. in Lake Geneva, Switzerland. Plastics were found on every beach and in the surface layer of Lake Geneva. However, no plastics were observed in biota in this study (Faure et al. 2012). Studies detecting microplastics in different freshwater compartments across continents and even in remote areas (Free et al. 2014; Zhang et al. 2016) are summarized in Tables 1-4.

The numbers of microplastic studies in freshwater environments increased rapidly from four in 2013 to 37 studies in 2019 and 27 papers as of September 2020 (Fig. 2).

In the early stages, it was suggested that the chemical types of microplastics in freshwater seem to be less diverse compared to those collected from the marine salty environment. This observation was attributed to the higher density of seawater, which enables more types of plastic materials with different densities to float on the surface of the water (Zhang et al. 2015). However, this is not always the case, because the processes of controlling distribution and exposure to plastics particles are not necessarily restricted to a specific environmental compartment. Polymers with higher density (density $>1.0 \mathrm{~g} \mathrm{~mL}^{-1}$ ) were observed in freshwater environments (Moore et al. 2011; Zhou et al. 2020). Negatively buoyant particles (e.g., polyester, rayon, nylon and cellulose acetate) may remain suspended in water (Baldwin et al. 2016). There may be differing degrees of physical and chemical characteristics, such as storms and wave action and saline water in marine systems. But plastics in freshwater systems still experience physical and chemical degradation (Andrady et al. 2011). It was suggested that polymer density alone is not the most significant control on microplastic particle fate within the aquatic environment. Microplastic morphology, incorporation into copolymeric materials during manufacturing and inclusion within aggregates of varying overall densities may play major roles in microplastics distribution (Hendrickson et al. 2018).

The ability to capture plastic particles from water or sediment matrix and separating them from organic and mineral material are challenging. Identifying types of plastics in the samples and on different surfaces is also important (Costa et al. 2021). It is suggested that microplastics in freshwater systems are similar to those in marine environments, and they are exposed to similar threats (Holland et al. 2016). Therefore, microplastic characteristics, detection methods, methods of analysis and impacts on biota are suggested to be similar.

\section{Sampling procedure and tools}

Choice of preservation techniques in different stages of microplastic studies largely depend on the research question (Lusher et al. 2017), economic proportionality of the methods and also the study compartment. Microplastics have now been reported in a range of freshwater environments, including surface water, water column, benthic sediments, littoral sediments and aquatic biota. Three main strategies are identified for sampling. They include selective sampling, volume-reduced sampling and bulk sampling. Different sampling strategies may be selected when the type of matrix to be examined for microplastics (water or sediment or biota) has been taken into account. Selective sampling in field consists of direct collection of items from the environment which are recognizable by the naked eye. This method is usually used on the surface of shore sediments and is more practical for large microplastics (1-5 mm). Bulk samples refer to samples where the whole volume of the sample is taken without reducing it during the sampling process. Volume-reduced samples in both sediment and water samples refer to samples where the volume of the bulk sample is usually reduced during sampling. Only a portion of the sample is preserved in this method, and it is mostly used for water samples (HidalgoRuz et al. 2012).

\section{Water compartment}

In water samples, microplastic burden in the measuring units is much lower compared to that in sediment samples. Consequently, analysis of water samples requires higher sampling volumes (Huppertsberg and Knepper 2018). Volume-reduced methods are on-site filtration by nets or sieving. They are more suitable for water samples as they give a promising specimen volume without the need to transfer the whole to the laboratory. Therefore, resulting in a relatively small concentrated final sample. Here we discus three main water sampling methods, including trawls, pump samplers and grab samples.

\section{Trawls and nets}

Different types of trawls and nets like manta, neuston or plankton nets and bongo net are used (Fig. 3). Trawl is usually deployed off of a boat, submerged and towed on a linear course at a low speed for a set time or distance (Hidalgo-Ruz et al. 2012; Sighicelli et al. 2018). The area of each sampling is calculated by multiplying the towing distance with the width of the trawl. The volume of water through the net uses either a flow meter or calculations based on the distance traveled by boat at a constant speed 
Table 1 Microplastic studies in water of inland water bodies around the world

\begin{tabular}{|c|c|c|c|c|c|}
\hline $\begin{array}{l}\text { Study } \\
\text { compartment }\end{array}$ & Number & Study area & Sampling tools & $\begin{array}{l}\text { Dominant microplastic } \\
\text { characteristics (shape, polymer } \\
\text { type, size) }\end{array}$ & Reference \\
\hline \multirow[t]{13}{*}{ River-estuary } & 1 & Three Gorges Dam-China & $\begin{array}{l}\text { Trawl with a rectangular } \\
\text { opening } 50 \mathrm{~cm} \text { high by } \\
100 \mathrm{~cm} \text { wide, and } 1.5 \mathrm{~m} \\
\text { long, } 112-\mathrm{mm} \text {-mesh size } \\
\text { nylon net with a } 500-\mathrm{mL} \\
\text { polyethylene collecting } \\
\text { bottle at the end }\end{array}$ & Sheets, PP, $500 \mu \mathrm{m}-1.6 \mathrm{~mm}$ & $\begin{array}{l}\text { Zhang et al. } \\
\text { (2015) }\end{array}$ \\
\hline & 2 & $\begin{array}{l}\text { The North Shore Channel } \\
\text { (NSC) in Chicago, Illinois } \\
\text { (IL), USA }\end{array}$ & $\begin{array}{l}\text { Two neuston nets } \\
(0.92 \times 0.42 \mathrm{~m} \text { and } \\
0.36 \times 0.41 \mathrm{~m}), 333 \mu \mathrm{m} \\
\text { mesh size }\end{array}$ & Fiber & $\begin{array}{l}\text { McCormick } \\
\text { et al. } \\
\text { (2014) }\end{array}$ \\
\hline & 3 & $\begin{array}{l}29 \text { Great Lakes tributaries, } \\
\text { USA }\end{array}$ & $\begin{array}{l}\text { Neuston net } 1.5-\mathrm{m}-\text { long net } \\
\text { with an opening } 100 \mathrm{~cm} \\
\text { wide by } 40 \mathrm{~cm} \text { high, } \\
333 \mu \mathrm{m} \text { mesh size }\end{array}$ & Fibers, $0.355-0.999 \mathrm{~mm}$ & $\begin{array}{l}\text { Baldwin } \\
\text { et al. } \\
(2016)\end{array}$ \\
\hline & 4 & $\begin{array}{l}\text { Inflow (Red and Assiniboine } \\
\text { rivers) and outflow (Nelson } \\
\text { River) of LakeWinnipeg, } \\
\text { Canada }\end{array}$ & $\begin{array}{l}\text { Manta trawl } 295 \mathrm{~cm} \text { long, an } \\
\text { aperture width of } 61 \mathrm{~cm}, \\
\text { and a heightof } 18 \mathrm{~cm}, \\
333 \mu \mathrm{m} \text { mesh size }\end{array}$ & Fibers & $\begin{array}{l}\text { Warrack } \\
\text { et al. } \\
(2018)\end{array}$ \\
\hline & 5 & Tamar Estuary, UK & $\begin{array}{l}\text { Manta net } 0.50 \mathrm{~m} \text { by } 0.15 \mathrm{~m} \text {, } \\
300 \mu \mathrm{m} \text { mesh size }\end{array}$ & Fragments, PE, 1-3 mm & $\begin{array}{l}\text { Sadri and } \\
\text { Thompson } \\
\text { (2014) }\end{array}$ \\
\hline & 6 & $\begin{array}{l}\text { Los Angeles and San Gabriel } \\
\text { Rivers, USA }\end{array}$ & $\begin{array}{l}\text { Manta trawl } 0.9 \mathrm{~m} \times 0.15 \mathrm{~m} \text {, } \\
333 \mu \mathrm{m} \text { mesh size, hand } \\
\text { nets } 0.46 \mathrm{~m} \times 0.25 \mathrm{~m}, \\
800 \mu \mathrm{m} \text { mesh size and } \\
0.43 \mathrm{~m} \times 0.22 \mathrm{~m}, 500 \mu \mathrm{m} \\
\text { mesh size, streambed } \\
0.15 \mathrm{~m} \times 0.15 \mathrm{~m}, 333 \\
\text { mesh size, rectangular net } \\
0.45 \mathrm{~m} \times 0.25 \mathrm{~m}, 333 \\
\text { mesh size }\end{array}$ & $\begin{array}{l}\text { Foams, PS, } \geq 1 \mathrm{~mm} \\
\text { and }<4.75 \mathrm{~mm}\end{array}$ & $\begin{array}{l}\text { Moore et al. } \\
\text { (2011) }\end{array}$ \\
\hline & 7 & Danube River, Austria & $\begin{array}{l}\text { Stationary conical drift nets } \\
0.5 \mathrm{~m} \text { diameter, } 1.5 \mathrm{~m} \text { long, } \\
500 \mu \mathrm{m} \text { mesh size }\end{array}$ & - & $\begin{array}{l}\text { Lechner } \\
\text { et al. } \\
\text { (2014) }\end{array}$ \\
\hline & 8 & $\begin{array}{l}\text { Four Estuarine Rivers in the } \\
\text { Chesapeake Bay ( Patapsco, } \\
\text { Magothy, Rhode, and } \\
\text { Corsica rivers), USA }\end{array}$ & $\begin{array}{l}\text { Manta net } 70 \mathrm{~cm} \text { wide, } \\
330 \mu \mathrm{m} \text { mesh size }\end{array}$ & - & $\begin{array}{l}\text { Yonkos et al. } \\
\text { (2014) }\end{array}$ \\
\hline & 9 & $\begin{array}{l}\text { Yangtze Estuary and East } \\
\text { China Sea, China }\end{array}$ & $\begin{array}{l}\text { Teflon pump passed through a } \\
32-\mu \mathrm{m} \text { steel sieve, neuston } \\
\text { trawl } 30 \times 40 \mathrm{~cm}^{2} \text { opening, } \\
333 \mu \mathrm{m} \text { mesh size }\end{array}$ & Fibers, $>0.5-1 \mathrm{~mm}$ & $\begin{array}{l}\text { Zhao et al. } \\
\text { (2014) }\end{array}$ \\
\hline & 10 & Rhine River-Switzerland & $\begin{array}{l}\text { Manta trawl with rectangular } \\
\text { opening of } 60 \mathrm{~cm} \times 18 \mathrm{~cm} \text {, } \\
\text { mesh } 300 \mu \mathrm{m} \text { mesh size }\end{array}$ & Opaque spherules, PS & $\begin{array}{l}\text { Mani et al. } \\
\text { (2015) }\end{array}$ \\
\hline & 11 & $\begin{array}{l}\text { Solent estuarine complex ( } \\
\text { Hamble, Itchen and Test } \\
\text { rivers), UK }\end{array}$ & $\begin{array}{l}\text { Plankton net trawl, } 300 \mu \mathrm{m} \\
\text { mesh size }\end{array}$ & $\begin{array}{l}\text { Fibers, blue, black, clear, } \\
\text { white }\end{array}$ & $\begin{array}{l}\text { Gallagher } \\
\text { et al. } \\
(2016)\end{array}$ \\
\hline & 12 & $\begin{array}{l}\text { Three urban estuaries ( } \\
\text { Jiaojiang, Minjiang and } \\
\text { Oujiang Estuaries), China }\end{array}$ & $\begin{array}{l}\text { Teflon pump passed through a } \\
333-\mu \mathrm{m} \text { steel sieve }\end{array}$ & $\begin{array}{l}\text { Fibers, } \\
\qquad \mathrm{PP},<0.5-1 \mathrm{~mm},<1-2 \mathrm{~mm}\end{array}$ & $\begin{array}{l}\text { Zhao et al. } \\
\text { (2015) }\end{array}$ \\
\hline & 13 & $\begin{array}{l}\text { Pearl River along Guangzhou } \\
\text { city and Pearl River estuary, } \\
\text { China }\end{array}$ & $\begin{array}{l}\text { Water sampler passed through } \\
\text { a } 50-\mu \mathrm{m} \text { stainless steel sieve }\end{array}$ & Films, PA, $<0.5 \mathrm{~mm}$ & $\begin{array}{l}\text { Yan et al. } \\
\text { (2019) }\end{array}$ \\
\hline
\end{tabular}


Table 1 (continued)

\begin{tabular}{|c|c|c|c|c|c|}
\hline $\begin{array}{l}\text { Study } \\
\text { compartment }\end{array}$ & Number & Study area & Sampling tools & $\begin{array}{l}\text { Dominant microplastic } \\
\text { characteristics (shape, polymer } \\
\text { type, size) }\end{array}$ & Reference \\
\hline & 14 & Saigon River, Vietnam & $\begin{array}{l}\text { A bucket and plankton net, } \\
300 \mu \mathrm{m} \text { mesh size }\end{array}$ & Fibers, PES & $\begin{array}{l}\text { Lahens et al. } \\
\text { (2018) }\end{array}$ \\
\hline & 15 & Hudson River, USA & $\begin{array}{l}\text { Grab sample, metal bucket } \\
(3 \mathrm{~L})\end{array}$ & Fibers, PET & $\begin{array}{l}\text { Miller et al. } \\
\text { (2017) }\end{array}$ \\
\hline & 16 & $\begin{array}{l}\text { Raritan River, New Jersey, } \\
\text { USA }\end{array}$ & $\begin{array}{l}\text { Plankton nets } 0.2 \mathrm{~m} \text { diameter, } \\
0.51 \mathrm{~m} \text { long, } 153 \mathrm{~mm} \text { mesh } \\
\text { size }\end{array}$ & - & $\begin{array}{l}\text { Estahbanati } \\
\text { and } \\
\text { Fahrenfeld } \\
\text { (2016) }\end{array}$ \\
\hline & 17 & Rivers State, Nigeria & Plankton net & - & $\begin{array}{l}\text { Briggs et al. } \\
\text { (2019) }\end{array}$ \\
\hline & 18 & Meuse, Rhine, Europe & - & Fibers & $\begin{array}{l}\text { Brandsma } \\
\text { et al. } \\
\text { (2013) }\end{array}$ \\
\hline & 19 & $\begin{array}{c}\text { Goose Creek, Little Kickapoo } \\
\text { Creek, and East Branch of } \\
\text { the DuPage River, USA }\end{array}$ & $\begin{array}{l}\text { Neuston nets } \\
0.52 \mathrm{~m} \times 0.36 \mathrm{~m}, 333 \mu \mathrm{m} \\
\text { mesh size }\end{array}$ & Pellets, PE & $\begin{array}{l}\text { McCormick } \\
\text { et al. } \\
(2016)\end{array}$ \\
\hline & 20 & $\begin{array}{l}\text { Rhine, Dalålven, Danube and } \\
\text { Po Rivers, Europe }\end{array}$ & $\begin{array}{l}\text { Manta net internal width } \\
60 \mathrm{~cm}, 330 \mu \mathrm{m} \text { mesh size, } \\
\text { Pump, waste free water } \\
\text { sampler mesh size } 3.2 \mathrm{~mm}\end{array}$ & Fragments, pellets, PE & $\begin{array}{l}\text { van der Wal } \\
\text { et al. } \\
\text { (2015) }\end{array}$ \\
\hline & 21 & $\begin{array}{l}\text { Jade system, south North Sea, } \\
\text { Germany }\end{array}$ & $\begin{array}{l}\text { Grab sample, PE bottle } \\
(100 \mathrm{ml}) \text { passed through } \\
1.2-\mu \mathrm{m} \text { cellulose nitrate } \\
\text { filters }\end{array}$ & Fibers & $\begin{array}{l}\text { Dubaish and } \\
\text { Liebezeit } \\
\text { (2013) }\end{array}$ \\
\hline & 22 & $\begin{array}{l}\text { Snake River and Palisades } \\
\text { Reservoir, USA }\end{array}$ & - & Fibers & $\begin{array}{l}\text { McDevitt } \\
\text { and Perez } \\
(2016)\end{array}$ \\
\hline & 23 & 29 Rivers, Japan & $\begin{array}{l}\text { Plankton net } 30 \mathrm{~cm} \times 75 \mathrm{~cm} \text {, } \\
335 \mu \mathrm{m} \text { mesh size }\end{array}$ & - & $\begin{array}{l}\text { Kataoka } \\
\text { et al. } \\
(2019)\end{array}$ \\
\hline & 24 & $\begin{array}{l}\text { Snake River and Columbia } \\
\text { River, USA }\end{array}$ & $\begin{array}{l}\text { Grab sample, glass jars, mean } \\
\text { volume } 1.85 \mathrm{~L} \text {, plankton } \\
\text { net, } 100 \mu \mathrm{m} \text { mesh size }\end{array}$ & Fibers, $100-333 \mu \mathrm{m}$ & $\begin{array}{l}\text { Kapp and } \\
\text { Yeatman } \\
(2018)\end{array}$ \\
\hline & 25 & Rhine River, Germany & $\begin{array}{l}\text { Manta trawl } 60 \mathrm{~cm} \times 18 \mathrm{~cm} \\
\text { rectangular aperture, } \\
300 \mu \mathrm{m} \text { mesh size }\end{array}$ & PS-DVB ion-exchange resins & $\begin{array}{l}\text { Mani et al. } \\
\text { (2019a) }\end{array}$ \\
\hline & 26 & $\begin{array}{l}\text { Gallatin River watershed, } \\
\text { USA }\end{array}$ & $\begin{array}{l}\text { Grab sample, stainless steel } \\
\text { bottles }(1 \mathrm{~L})\end{array}$ & $\begin{array}{l}\text { Fibers, Semi-synthetic } \\
\text { cellulose, PES, 0.1-1.5 mm }\end{array}$ & $\begin{array}{l}\text { Barrows } \\
\text { et al. } \\
(2018)\end{array}$ \\
\hline & 27 & Changjiang Estuary, China & $\begin{array}{l}\text { A screw pump }(100 \mathrm{~L}) \text { passed } \\
\text { through a stainless steel } \\
\text { sieve } 60 \mu \mathrm{m} \text { pore size }\end{array}$ & Fiber, PE & $\begin{array}{l}\text { Zhao et al. } \\
\text { (2019) }\end{array}$ \\
\hline & 28 & Changjiang Estuary, China & $\begin{array}{l}\text { A pump (100 L) passed } \\
\text { through stainless steel sieve } \\
70 \mu \mathrm{m} \text { mesh size }\end{array}$ & Fibers, PE0.07-1.0 mm & $\begin{array}{c}\text { Xu et al. } \\
(2018)\end{array}$ \\
\hline & 29 & Pasig River, Philippines & $\begin{array}{l}\text { Two Manta trawl } 25.7 \mathrm{~cm} \\
\text { diameter openingand } \\
10.4 \mathrm{~cm} \text { diameter opening, } \\
355 \mu \mathrm{m} \text { mesh size }\end{array}$ & Fragments, $1.16 \pm 0.42 \mathrm{~mm}$ & $\begin{array}{l}\text { Deocaris } \\
\text { et al. } \\
(2019)\end{array}$ \\
\hline & 30 & Danube River, Austria & $\begin{array}{l}\text { Net } 600 \times 600 \mathrm{~mm} \text { opening, } \\
500,250,41 \mu \mathrm{m} \text { mesh size, } \\
\text { BFG basket sampler } \\
300 \times 600 \mathrm{~mm} \text { opening, } \\
500 \mu \mathrm{m} \text { mesh size }\end{array}$ & - & $\begin{array}{l}\text { Liedermann } \\
\text { et al. }(2018\end{array}$ \\
\hline
\end{tabular}


Table 1 (continued)

\begin{tabular}{|c|c|c|c|c|c|}
\hline $\begin{array}{l}\text { Study } \\
\text { compartment }\end{array}$ & Number & Study area & Sampling tools & $\begin{array}{l}\text { Dominant microplastic } \\
\text { characteristics (shape, polymer } \\
\text { type, size) }\end{array}$ & Reference \\
\hline & 31 & $\begin{array}{l}\text { Muskegon River, Milwaukee } \\
\text { River, and St. Joseph River, } \\
\text { USA }\end{array}$ & $\begin{array}{l}\text { Grab samples, } 2 \text {-L glass bottle } \\
\text { passed through a } 0.363 \mu \mathrm{m} \\
\text { mesh (surfaces water), } \\
\text { Wading seine nets (biota) }\end{array}$ & Fibers, $<1.5 \mathrm{~mm}$ & $\begin{array}{l}\text { McNeish } \\
\text { et al. } \\
\text { (2018) }\end{array}$ \\
\hline & 32 & $\begin{array}{l}\text { Clyde, Bega and Hunter } \\
\text { estuaries, Australia }\end{array}$ & $\begin{array}{l}\text { Horizontal surface tows using } \\
45 \mu \mathrm{m} \text { mesh size (biota), } \\
\text { vertical towl } 37 \mu \mathrm{m} \text { net } \\
\text { (biota) }\end{array}$ & Fragments, 45-100 $\mu \mathrm{m}$ & $\begin{array}{l}\text { Hitchcock } \\
\text { and } \\
\text { Mitrovic } \\
\text { (2019) }\end{array}$ \\
\hline & 33 & Douro estuary, Portugal & $\begin{array}{l}\text { A conical } 1 \mathrm{~m} \text { diameter, } 4 \mathrm{~m} \\
\text { long, } 500 \mu \mathrm{m} \text { mesh size } \\
\text { (biota) }\end{array}$ & - & $\begin{array}{l}\text { Rodrigues } \\
\text { et al. } \\
\text { (2019) }\end{array}$ \\
\hline & 34 & Ofanto River, Italy & $\begin{array}{l}\text { Plankton nets } 2.5 \mathrm{~m} \text { long with } \\
\text { an opening of } \\
55 \mathrm{~cm} \times 55 \mathrm{~cm}, 333 \mu \mathrm{m} \\
\text { mesh size }\end{array}$ & $\begin{array}{l}\text { Fragments, flakes, PE, } \\
\text { 500-1000 } \mu \mathrm{m}, \\
1000-2000 \mu \mathrm{m}\end{array}$ & $\begin{array}{l}\text { Campanale } \\
\text { et al. } \\
(2020)\end{array}$ \\
\hline & 35 & Yellow River, China & Stainless steel bucket (5 L) & Fibers, $50-100 \mu \mathrm{m}$ & $\begin{array}{l}\text { Han et al. } \\
\qquad(2020)\end{array}$ \\
\hline & 36 & $\begin{array}{l}\text { Swiss Rhine River catchment } \\
\text { at Brugg and the } \\
\text { downstream German-Dutch } \\
\text { border at Rees (Germany } \\
\text { and Switzerland) }\end{array}$ & $\begin{array}{l}\text { Manta trawl } 60 \mathrm{~cm} \times 18 \mathrm{~cm} \text {, } \\
300 \mu \mathrm{m} \text { mesh size }\end{array}$ & Fragments, PE, 0.3-1 mm & $\begin{array}{l}\text { Mani and } \\
\text { Burkhardt- } \\
\text { Holm } \\
(2020)\end{array}$ \\
\hline & 37 & $\begin{array}{l}\text { Urban waters of seven cities in } \\
\text { the Tuojiang River basin, } \\
\text { China }\end{array}$ & $\begin{array}{l}\text { Steel sampler }(25 \mathrm{~L}) \text { passed } \\
\text { through } 50 \mu \mathrm{m} \text { mesh size } \\
\text { sieve }\end{array}$ & Fibers, PP, 0.5-1 mm & $\begin{array}{l}\text { Zhou et al. } \\
\qquad(2020)\end{array}$ \\
\hline & 38 & Manas River, China & Stainless steel drum (2.5 L) & Fibers, PP, $0.3-1.0 \mathrm{~mm}$ & $\begin{array}{l}\text { G. Wang } \\
\text { et al. } \\
\text { (2020) }\end{array}$ \\
\hline & 39 & $\begin{array}{l}\text { Minjiang River watershed, } \\
\text { Southeast China }\end{array}$ & $\begin{array}{l}\text { Metal pail passed through } \\
300 \mu \mathrm{m} \text { mesh size }\end{array}$ & Fibers, PET, 1-2 mm & $\begin{array}{l}\text { Huang et al. } \\
\text { (2020) }\end{array}$ \\
\hline & 40 & $\begin{array}{l}\text { Meuse river and in } \\
\text { Netherlands and the } \\
\text { Dommel, Germany }\end{array}$ & $\begin{array}{l}\text { A centrifugal water pump } \\
\text { passed through of } 300,100, \\
\text { and } 20 \mu \mathrm{m} \text { mesh size sieve }\end{array}$ & PE, 0-1000 $\mu \mathrm{m}$ & $\begin{array}{l}\text { Mintenig } \\
\text { et al. } \\
\text { (2020) }\end{array}$ \\
\hline & 41 & $\begin{array}{l}\text { Cherating river and mangrove, } \\
\text { Malaysia }\end{array}$ & $\begin{array}{l}\text { Conical nylon plankton net } \\
0.3 \mathrm{~m} \text { x } 1 \mathrm{~m}, 100 \mu \mathrm{m} \text { mesh } \\
\text { size }\end{array}$ & Fragments, $0.5-1.0 \mathrm{~mm}$ & $\begin{array}{l}\text { Pariatamby } \\
\text { et al. } \\
(2020)\end{array}$ \\
\hline & 42 & Yulin River, China & $\begin{array}{l}\text { Teflon pump }\left(0.05 \mathrm{~m}^{3}\right) \\
\text { passed through } 64 \mu \mathrm{m} \\
\text { stainless steel sieve mesh } \\
\text { size }\end{array}$ & Lines/fibers, PE, 64-100 $\mu \mathrm{m}$ & $\begin{array}{l}\text { Y. Mao et al. } \\
\text { (2020) }\end{array}$ \\
\hline & 43 & Qing River, Beijing, China & $\begin{array}{l}\text { Stainless steel bucket }(20 \mathrm{~L}) \\
\text { passed through stainless } \\
\text { steel } 5000 \mu \mathrm{m} \text { mesh size }\end{array}$ & Fragments, PE, EPR & $\begin{array}{l}\text { C. Wang } \\
\text { et al. } \\
(2020)\end{array}$ \\
\hline \multirow[t]{3}{*}{ Lake-reservoir } & 1 & $\begin{array}{l}3 \text { connected urban lakes and } \\
\text { drainage playa wetlands, } \\
\text { Lubbock, Texas, USA }\end{array}$ & $\begin{array}{l}\text { Grab sample }(3.50 \mathrm{~L}) \text { passed } \\
\text { through sieve }(>300, \\
250-299,180-249, \\
106-179, \text { and } 53-105 \mu \mathrm{m} \\
\text { mesh size }\end{array}$ & $53-105 \mu \mathrm{m}$ & $\begin{array}{l}\text { Lasee et al. } \\
\text { (2017) }\end{array}$ \\
\hline & 2 & $\begin{array}{l}\text { Lake Hovsgol (mountain } \\
\text { remote lake), Mongolia }\end{array}$ & $\begin{array}{l}\text { Manta trawl } 16 \mathrm{~cm} \\
\text { high } \times 61 \mathrm{~cm} \text { wide and a } \\
3 \mathrm{~m} \text { long, } 333 \mu \mathrm{m} \text { mesh size }\end{array}$ & $\begin{array}{l}\text { Lines/fibers, } 0.355-0.999 \mathrm{~mm} \text {, } \\
1.00-4.749 \mathrm{~mm}\end{array}$ & $\begin{array}{l}\text { Free et al. } \\
\quad(2014)\end{array}$ \\
\hline & 3 & $\begin{array}{l}\text { Laurentian Great Lakes ( } \\
\text { Lakes Superior, Huron and } \\
\text { Erie), USA }\end{array}$ & $\begin{array}{l}\text { Manta trawl with a } \\
\text { rectangular opening } 16 \mathrm{~cm} \\
\text { high } \times 61 \mathrm{~cm} \text { wide, and a } \\
3 \mathrm{~m} \text { long, } 333 \mu \mathrm{m} \text { mesh size }\end{array}$ & Pellets, 0.355-0.999 mm & $\begin{array}{l}\text { Eriksen et al. } \\
\text { (2013) }\end{array}$ \\
\hline
\end{tabular}


Table 1 (continued)

\begin{tabular}{|c|c|c|c|c|c|}
\hline $\begin{array}{l}\text { Study } \\
\text { compartment }\end{array}$ & Number & Study area & Sampling tools & $\begin{array}{l}\text { Dominant microplastic } \\
\text { characteristics (shape, polymer } \\
\text { type, size) }\end{array}$ & Reference \\
\hline & 4 & Lake Winnipeg, Canada & $\begin{array}{l}\text { Manta } 61 \mathrm{~cm} \text { wide } \times 18 \mathrm{~cm} \\
\text { high and a } 3 \text { long, } 333 \mu \mathrm{m} \\
\text { mesh size }\end{array}$ & Fibers & $\begin{array}{l}\text { Anderson } \\
\text { et al. } \\
\text { (2017) }\end{array}$ \\
\hline & 5 & $\begin{array}{l}\text { Dongting Lake and Hong } \\
\text { Lake, China }\end{array}$ & $\begin{array}{l}12 \mathrm{~V} \text { DC Teflon pump }(20 \mathrm{~L}) \\
\text { passed through } 50 \mu \mathrm{m} \text { mesh } \\
\text { size }\end{array}$ & $\begin{array}{l}\text { Fibers, PP, PE, 50-330 } \mu \mathrm{m} \text {, } \\
\text { 330-1000 } \mathrm{mm}\end{array}$ & $\begin{array}{l}\text { W.F. Wang } \\
\text { et al. } \\
\text { (2018) }\end{array}$ \\
\hline & 6 & Western Lake Superior, USA & $\begin{array}{l}\text { Manta net } 85 \mathrm{~cm} \\
\text { wide } \times 14 \mathrm{~cm} \text { high and a } \\
3 \mathrm{~m} \text { long, } 333 \mu \mathrm{m} \text { mesh size }\end{array}$ & Fibers, PVC & $\begin{array}{l}\text { Hendrickson } \\
\text { et al. } \\
\text { (2018) }\end{array}$ \\
\hline & 7 & Lake Michigan, USA & $\begin{array}{l}\text { Manta trawl } 61 \mathrm{~cm} \\
\text { wide } \times 16 \mathrm{~cm} \text { high and a } \\
3 \mathrm{~m} \text { long, } 333 \mu \mathrm{m} \text { mesh size }\end{array}$ & $\begin{array}{l}\text { Fragments, PE, 0.355- } \\
.999 \mathrm{~mm}\end{array}$ & $\begin{array}{l}\text { Mason et al. } \\
\text { (2016) }\end{array}$ \\
\hline & 8 & $\begin{array}{l}\text { Lake Maggiore, Iseo and } \\
\text { Garda, Italy }\end{array}$ & $\begin{array}{l}\text { Manta trawl } 60 \times 20 \mathrm{~cm}, \\
300 \mu \mathrm{m} \text { mesh size }\end{array}$ & Fragments, PE & $\begin{array}{l}\text { Sighicelli } \\
\text { et al. } \\
\text { (2018) }\end{array}$ \\
\hline & 9 & $\begin{array}{l}\text { Urban Lakes in Changsha, } \\
\text { China }\end{array}$ & $\begin{array}{l}40 \mathrm{~L} \text { water passed through } \\
45 \mu \mathrm{m} \text { mesh size }\end{array}$ & Lines, 50-500 $\mu \mathrm{m}$ & $\begin{array}{l}\text { Yin et al. } \\
\text { (2019) }\end{array}$ \\
\hline & 10 & $\begin{array}{l}\text { Feilaixia Reservoir in the } \\
\text { Beijiang River, China }\end{array}$ & $\begin{array}{l}\text { Conical plankton } 20 \mathrm{~cm} \\
\text { diameter, } 112 \mu \mathrm{m} \text { mesh size }\end{array}$ & Films, PP, 0.6-2 mm & $\begin{array}{l}\text { Tan et al. } \\
\text { (2019) }\end{array}$ \\
\hline & 11 & Lake Ulansuhai, China & $\begin{array}{l}\text { 12-V DC Teflon pump passed } \\
\text { through } 48 \mu \mathrm{m} \text { mesh size }\end{array}$ & Fibers, PE, $<0.5 \mathrm{~mm}$ & $\begin{array}{l}\text { Wang et al. } \\
\quad(2019\end{array}$ \\
\hline & 12 & $\begin{array}{l}\text { Mecklenburg Lake District in } \\
\text { Mecklenburg-Western } \\
\text { Pomerania, Germany }\end{array}$ & $\begin{array}{l}\text { Pump water samples, Manta } \\
\text { trawl }\end{array}$ & $\begin{array}{l}\text { Irregular particles, PE, PET, } \\
0-1000 \mu \mathrm{m}\end{array}$ & $\begin{array}{l}\text { Tamminga } \\
\text { et al. } 2020\end{array}$ \\
\hline & 13 & $\begin{array}{l}\text { Wuliangsuhai Lake, northern } \\
\text { China }\end{array}$ & $\begin{array}{l}\text { Stainless steel buckets }(20 \mathrm{~L}) \\
\text { passed through } 75 \mu \mathrm{m} \text { mesh } \\
\text { size sieve }\end{array}$ & Fibers, PS, $<0.5 \mathrm{~mm}$ & $\begin{array}{l}\text { R. Mao et al. } \\
\text { (2020) }\end{array}$ \\
\hline Stream & 1 & $\begin{array}{l}\text { Six Mile Creek and Fall Creek } \\
\text { streams, USA }\end{array}$ & $\begin{array}{l}\text { Neuston net } \\
1 \times 0.5 \mathrm{~m}, 335 \mu \mathrm{m} \text { mesh } \\
\text { size }\end{array}$ & Fibers & $\begin{array}{l}\text { Watkins } \\
\text { et al. } \\
\text { (2019b) }\end{array}$ \\
\hline Stream-lake & 1 & $\begin{array}{l}\text { Streams and wetlands, } \\
\text { Victoria, Australia }\end{array}$ & $\begin{array}{l}\text { Grab surface, polypropylene } \\
\text { jars (5L) (water), dip nets } \\
\text { (biota) }\end{array}$ & Fibers, PES, rayon, 0-1 mm & $\begin{array}{l}\text { Nan et al. } \\
\quad(2020)\end{array}$ \\
\hline Pond & 1 & North of Jutland, Denmark & $\begin{array}{l}\text { A positive displacement pump } \\
\text { passed through } 10 \mu \mathrm{m} \\
\text { stainless steel mesh size }\end{array}$ & PP & $\begin{array}{l}\text { Liu et al. } \\
\text { (2019) }\end{array}$ \\
\hline $\begin{array}{l}\text { River-estuary- } \\
\text { lake }\end{array}$ & 1 & $\begin{array}{l}\text { Urban lakes and urban reaches } \\
\text { of the Hanjiang River and } \\
\text { YangtzeRiver, Wuhan, } \\
\text { China }\end{array}$ & $\begin{array}{l}12 \mathrm{~V} \text { DC Teflon pump }(20 \mathrm{~L}) \\
\text { passed through } 50 \mu \mathrm{m} \\
\text { stainless steel sieve }\end{array}$ & $\begin{array}{l}\text { Fibers, PET, } 50-500 \mu \mathrm{m} \\
\quad \text { (or }<0.5 \mathrm{~mm} \text { ) }\end{array}$ & $\begin{array}{l}\text { W.F. Wang } \\
\text { et al. } \\
\text { (2017) }\end{array}$ \\
\hline $\begin{array}{l}\text { City creeks- } \\
\text { rivers-estuary } \\
\text { and coastal } \\
\text { waters }\end{array}$ & 1 & $\begin{array}{l}\text { City creeks (Shanghai), rivers } \\
\text { (Suzhou River and Huangpu } \\
\text { River), an estuary (Yangtze } \\
\text { Estuary) and coastal waters } \\
\text { (East China Sea), Yangtze } \\
\text { Delta area, China }\end{array}$ & $\begin{array}{l}\text { Metal pail }(5 \mathrm{~L}) \text { passed } \\
\text { through } 20 \mu \mathrm{m} \text { mesh size } \\
\text { filter, air lift pump }\end{array}$ & Fibers, PES, 0.1-1.0 mm & $\begin{array}{l}\text { Luo et al. } \\
\text { (2019) }\end{array}$ \\
\hline $\begin{array}{l}\text { River water- } \\
\text { wastewater- } \\
\text { total } \\
\text { atmospheric } \\
\text { fallout }\end{array}$ & 1 & $\begin{array}{l}\text { Greater Paris-Seine River, } \\
\text { France }\end{array}$ & $\begin{array}{l}\text { Manta trawl } 330 \mu \mathrm{m} \text { mesh } \\
\text { size, Plankton net } 80 \mu \mathrm{m} \\
\text { mesh size }\end{array}$ & Fibers, 1001-5000 $\mu \mathrm{m}$ & $\begin{array}{l}\text { Dris et al. } \\
(2015)\end{array}$ \\
\hline
\end{tabular}


Table 1 (continued)

\begin{tabular}{|c|c|c|c|c|c|}
\hline $\begin{array}{l}\text { Study } \\
\text { compartment }\end{array}$ & Number & Study area & Sampling tools & $\begin{array}{l}\text { Dominant microplastic } \\
\text { characteristics (shape, polymer } \\
\text { type, size) }\end{array}$ & Reference \\
\hline $\begin{array}{l}\text { River- } \\
\text { atmospheric } \\
\text { fallout-urban } \\
\text { runoff-WWTP } \\
\text { effluents-CSOs }\end{array}$ & 1 & River Marne, France & $\begin{array}{l}\text { Manta trawl } 80 \text { and } 300 \mu \mathrm{m} \\
\text { mesh size }\end{array}$ & Fibers & $\begin{array}{l}\text { Dris et al. } \\
\text { (2018) }\end{array}$ \\
\hline $\begin{array}{l}\text { Surface water, } \\
\text { storm water } \\
\text { runoff, } \\
\text { agricultural } \\
\text { runoff, and } \\
\text { treated } \\
\text { wastewater } \\
\text { effluent }\end{array}$ & 1 & $\begin{array}{l}\text { Lake Ontario of the } \\
\text { Laurentian Great Lakes in } \\
\text { Canada }\end{array}$ & $\begin{array}{l}\text { Stainless steel bucket }(4 \mathrm{~L}) \\
\text { passed through } 10 \mu \mathrm{m} \text { mesh } \\
\text { size filter }\end{array}$ & Fibers & $\begin{array}{l}\text { Grbić et al. } \\
(2020)\end{array}$ \\
\hline $\begin{array}{l}\text { Urban prairie } \\
\text { creek }\end{array}$ & 1 & $\begin{array}{l}\text { Wascana Creek, northern } \\
\text { outskirts of Regina, Canada }\end{array}$ & $\begin{array}{l}\text { Conical net (water), seine } \\
\text { nets, gill nets, conventional } \\
\text { tackle, and minnow traps } \\
\text { (biota) }\end{array}$ & Fibers & $\begin{array}{l}\text { Campbell } \\
\text { et al. } \\
\text { (2017) }\end{array}$ \\
\hline
\end{tabular}

(Sadri and Thompson 2014). However, because the net's immersion depth changes constantly with waves, wind and boat movement, it is difficult to estimate the exact volume of water being filtered.

Campanale et al. (2020) collected microplastics by three surface plankton nets fixed in the middle of Ofanto River, in order to reduce the spatial and temporal variability (Campanale et al. 2020). In order to ensure that the most representative body of water is being sampled, factors such as time, location and length of trawls in relation to the strength of tides should be carefully considered. Furthermore, the trawling distance using nets varies depending on the abundance of floating microplastics. It should further consider whether the trawl direction with reference to the prevailing wind direction could have an effect on the abundance and size of particles captured within the trawl (Zhang et al. 2018). In these methods, nets are limited by a single mesh size that is sometimes clogged by suspended material (e.g., organic matter or phytoplankton) (Liedermann et al. 2018; Sadri and Thompson 2014).

Types of microplastics are closely related to the mesh size of tools used for specimen collection. For instance, smaller-sized mesh used in some studies could increase plastic particles of certain shapes (e.g., fibers) to a concentration several orders of magnitude higher than those collected using nets with a larger mesh size. On that account, the abundance of microplastics is largely underestimated by researchers who used a trawl for sample collection (Z.F.Wang et al. 2018b). By using manta trawl, a significant fraction of actual small microplastic particles is very likely to be underestimated because they might pass through the net.
Regarding the limitations of obtaining sufficient water volumes while avoiding net clogging, it was strongly recommended to use tandem nets with different mesh sizes. This helps to better characterize smaller microplastics (Anderson et al. 2017). Dris et al. (2018) had a 250-times higher probability of sampling fibers when using an 80- $\mu \mathrm{m}$ mesh compared to a 330- $\mu \mathrm{m}$ mesh (Dris et al. 2018). Double neuston net trawl (500 $\mu \mathrm{m}$ mesh size) was used as sampling tool in assessing microplastics in surface waters of Lake Superior. No difference was detected between the paired net samples, suggesting that single net sampling produces a representative estimate of microplastic particle condition within a body of water (Cox 2018). A comparison study was conducted between a manta net and a neuston net for microplastics in ocean surface water. Results showed that the manta net tended to have slightly higher densities of microplastics than those of the neuston net. However, no statistical difference was observed. Neuston net is relatively stable in rough water although efforts are needed to maintain the net in submerged depth. Manta net tends to jump in rough water (Michida et al. 2019). Sampling with the manta trawl to function properly requires relatively calm conditions (Anderson et al. 2017). Modified BfG basket sampler used for the Austrian Danube River, clearly showed the necessity of a strong and stable equipment carrier. The nets were positioned on the surface, in the middle of the water column, and at the bottom of the river and with different mesh sizes (Liedermann et al. 2018).

Nets alone may fail to deliver the overall pattern of microplastic pollution in an area, because there does not seem to be sufficiently retaining fibers and small microplastics. 
Table 2 Microplastic studies in sediment of inland water bodies around the world

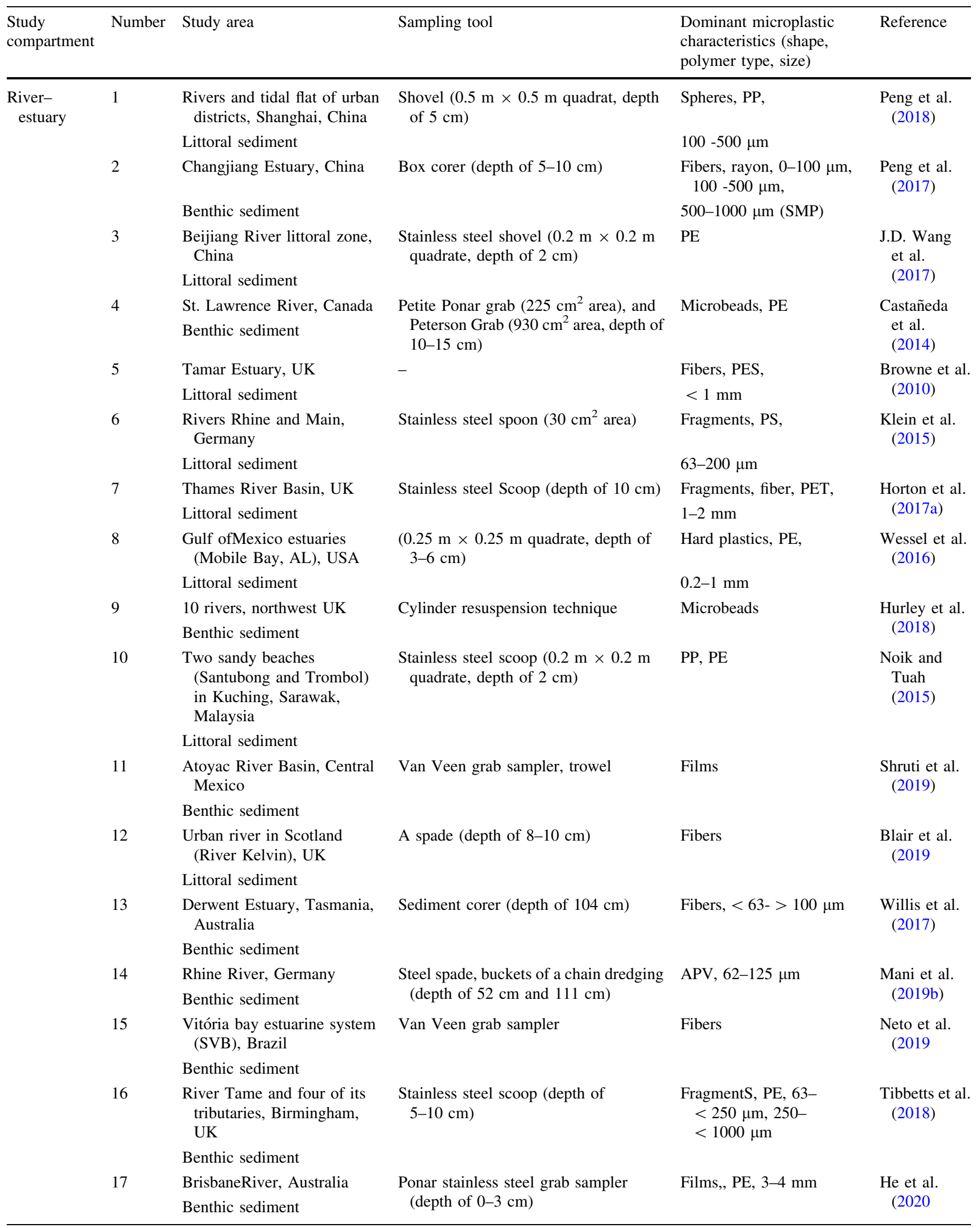


Table 2 (continued)

\begin{tabular}{|c|c|c|c|c|c|}
\hline $\begin{array}{l}\text { Study } \\
\text { compartment }\end{array}$ & Number & Study area & Sampling tool & $\begin{array}{l}\text { Dominant microplastic } \\
\text { characteristics (shape, } \\
\text { polymer type, size) }\end{array}$ & Reference \\
\hline & 18 & $\begin{array}{l}\text { Liaohe estuary, Daliao River } \\
\text { and Shuangtaizi River }\end{array}$ & Steel grab sampler & Films, PE & $\begin{array}{c}\text { Xu et al. } \\
(2020)\end{array}$ \\
\hline & & Benthic sediment & & & \\
\hline & 19 & $\begin{array}{l}\text { Thames River, Ontario, } \\
\text { Canada }\end{array}$ & $\begin{array}{l}\text { Stainless steel petite ponar grab } \\
\text { sampler (depth of } 90-100 \mathrm{~cm} \text { ) }\end{array}$ & Pellets, PE, $1-5 \mathrm{~cm}$ & $\begin{array}{l}\text { Corcoran } \\
\text { et al. }\end{array}$ \\
\hline & & Benthic sediment & & & (2019) \\
\hline & 20 & $\begin{array}{l}\text { Warnow estuarine, Germany } \\
\text { Benthic sediment }\end{array}$ & Van Veen grab, Sediment trap & PS & $\begin{array}{l}\text { Enders et al. } \\
\text { (2019) }\end{array}$ \\
\hline & 21 & $\begin{array}{l}\text { River Yongfeng, China } \\
\text { Benthic sediment }\end{array}$ & Peterson Gravity Sampler & $\begin{array}{l}\text { Films, PE, } 200-500 \mu \mathrm{m} \text {, } \\
500-1000 \mu \mathrm{m}\end{array}$ & $\begin{array}{l}\text { Rao et al. } \\
\quad(2020)\end{array}$ \\
\hline & 22 & $\begin{array}{l}\text { Jagir Estuary, Surabaya City, } \\
\text { Indonesia }\end{array}$ & Ekman dredge sampler & $\begin{array}{l}\text { Lines/fibers, PES, small } \\
\text { MP }(1 \mu \mathrm{m}-1 \mathrm{~mm})\end{array}$ & $\begin{array}{l}\text { Firdaus et al. } \\
\text { (2020) }\end{array}$ \\
\hline & & Benthic sediment & & & \\
\hline \multirow[t]{2}{*}{ Stream } & 1 & $\begin{array}{l}\text { Seven water } \\
\text { streamssurrounding the } \\
\text { lagoon of Bizerte, Northern } \\
\text { Tunisia }\end{array}$ & $\begin{array}{l}\text { Stainless steel spatula } \\
(0.25 \mathrm{~m} \times 0.25 \mathrm{~m} \text { quadrate, depth of } \\
2-3 \mathrm{~cm})\end{array}$ & Fibers, PP & $\begin{array}{l}\text { Toumi et al. } \\
\text { (2019) }\end{array}$ \\
\hline & & Littoral sediment & & & \\
\hline \multirow[t]{17}{*}{$\begin{array}{l}\text { Lake- } \\
\text { reservoir }\end{array}$} & 1 & Subalpine lake Garda, Italy & - & $\mathrm{PE}$ & $\begin{array}{l}\text { Imhof et al. } \\
\text { (2013) }\end{array}$ \\
\hline & & Littoral sediment & & & \\
\hline & 2 & Lake Ontario, Canada & Glew gravity corer, shipek grab, ponar & Fragments, PE & Ballent et al. \\
\hline & & $\begin{array}{l}\text { Littoral sediment and Benthic } \\
\text { sediment }\end{array}$ & & & \\
\hline & 3 & Lake Ontario, Canada & Mini box corer (depth of $30 \mathrm{~cm}$ ) & Pellets, PE, $1-5 \mathrm{~cm}$ & Corcoran \\
\hline & & $\begin{array}{l}\text { Littoral sediment and Benthic } \\
\text { sediment }\end{array}$ & & & $\begin{array}{l}\text { et al. } \\
(2015)\end{array}$ \\
\hline & 4 & $\begin{array}{l}\text { Remote lakes in Tibet } \\
\text { plateau, China }\end{array}$ & $\begin{array}{l}\text { Shovel }(20 \mathrm{~cm} \times 20 \mathrm{~cm} \text { quadrate, } \\
\text { depth of } 2 \mathrm{~cm})\end{array}$ & $\mathrm{PP}, 1-5 \mathrm{~mm}$ & $\begin{array}{l}\text { Zhang et al. } \\
\text { (2016) }\end{array}$ \\
\hline & & Littoral sediment & & & \\
\hline & 5 & $\begin{array}{l}\text { Beaches of Lake Huron, } \\
\text { Canada }\end{array}$ & Stainless steel trowel & Pellets, PE, $<5 \mathrm{~mm}$ & $\begin{array}{l}\text { Zbyszewski } \\
\text { and }\end{array}$ \\
\hline & & Littoral sediment & & & $\begin{array}{l}\text { Corcoran } \\
\text { (2011) }\end{array}$ \\
\hline & 6 & $\begin{array}{l}\text { Great Lakes, North America } \\
\text { Lake Erie and St. Clair), } \\
\text { USA }\end{array}$ & Stainless steel trowel & Fragments, PE & $\begin{array}{l}\text { Zbyszewski } \\
\text { et al. } \\
\text { (2014) }\end{array}$ \\
\hline & & Littoral sediment & & & \\
\hline & 7 & $\begin{array}{l}\text { Edgbaston Pool, } \\
\text { Birmingham, UK }\end{array}$ & HTH gravity corer (depth of $10 \mathrm{~cm}$ ) & Fibers, Films & $\begin{array}{l}\text { Vaughan } \\
\text { et al. }\end{array}$ \\
\hline & & $\begin{array}{l}\text { Littoral sediment and Benthic } \\
\text { sediment }\end{array}$ & & & (2017) \\
\hline & 8 & $\begin{array}{l}\text { Setúbal Lake, Portugal } \\
\text { Littoral sediment }\end{array}$ & $\begin{array}{l}\text { Stainless steel shovel } \\
(0.25 \mathrm{~m} \times 0.25 \mathrm{~m} \text { quadrate, } 3 \mathrm{~cm} \\
\text { depth })\end{array}$ & Fragments & $\begin{array}{l}\text { Blettler et al. } \\
\text { (2017) }\end{array}$ \\
\hline & 9 & $\begin{array}{l}\text { Beaches of Lake Garda, Italy } \\
\text { Littoral sediment }\end{array}$ & Sediment cores (depth of $10 \mathrm{~cm}$ ) & $1-50 \mu \mathrm{m}$ & $\begin{array}{l}\text { Imhof et al. } \\
\text { (2018) }\end{array}$ \\
\hline & 10 & $\begin{array}{l}\text { Lake Erie, Canada } \\
\text { Littoral sediment and Benthic } \\
\text { sediment }\end{array}$ & $\begin{array}{l}\text { Shipek sediment grab sampler and } \\
\text { passive sediment trap, split spoon } \\
\text { sampler, Petite Ponar grab sampler }\end{array}$ & Fibers, PE & $\begin{array}{l}\text { Dean et al. } \\
\text { (2018) }\end{array}$ \\
\hline
\end{tabular}


Table 2 (continued)

\begin{tabular}{|c|c|c|c|c|c|}
\hline $\begin{array}{l}\text { Study } \\
\text { compartment }\end{array}$ & Number & Study area & Sampling tool & $\begin{array}{l}\text { Dominant microplastic } \\
\text { characteristics (shape, } \\
\text { polymer type, size) }\end{array}$ & Reference \\
\hline & 11 & $\begin{array}{l}\text { Subalpine Lake Garda, Italy } \\
\text { Littoral sediment }\end{array}$ & Sediment cores (depth of $5 \mathrm{~cm}$ ) & - & $\begin{array}{l}\text { Imhof et al. } \\
\text { (2016) }\end{array}$ \\
\hline & 12 & $\begin{array}{l}\text { Three Gorges Reservoir, } \\
\text { China }\end{array}$ & $\begin{array}{l}\text { Stainless steel Trowel } \\
\qquad(20 \mathrm{~cm} \times 20 \mathrm{~cm} \text {, depth of } 2 \mathrm{~cm})\end{array}$ & Sheet,PP, $1-5 \mathrm{~mm}$ & $\begin{array}{l}\text { Zhang et al. } \\
\text { (2019 }\end{array}$ \\
\hline & & Littoral sediment & & & \\
\hline & 13 & $\begin{array}{l}\text { Hampstead Pond (Lake), UK } \\
\text { Benthic sediment }\end{array}$ & Piston corer (depth of $212 \mathrm{~cm}$ ) & Fibers & $\begin{array}{l}\text { Turner et al. } \\
\text { (2019) }\end{array}$ \\
\hline & 14 & $\begin{array}{l}\text { Lake Mjøsa and Lake } \\
\text { Femunden, Norway } \\
\text { Benthic sediment }\end{array}$ & $\begin{array}{l}\text { Kajak-Brinkhurst sediment corer (depth } \\
\text { of } 3 \mathrm{~cm} \text { ), Van Veen grab (depth of } \\
10-15 \mathrm{~cm} \text { ) }\end{array}$ & $\begin{array}{l}\text { Fibers, PS, Small } \\
\text { microplastics }<1 \mathrm{~mm}\end{array}$ & $\begin{array}{l}\text { Lusher et al. } \\
\text { (2018) }\end{array}$ \\
\hline & 15 & $\begin{array}{l}\text { Lake Victoria, Uganda, } \\
\text { Africa } \\
\text { Littoral sediment and Benthic } \\
\text { sediment }\end{array}$ & $\begin{array}{l}\text { Stainless steel trowel } \\
(0.5 \mathrm{~cm} \times 0.5 \mathrm{~cm} \text { quadrat, depth of } \\
5 \mathrm{~cm}) \text {, Ponar grab }\end{array}$ & $\begin{array}{l}\text { Films (shoreline), filaments } \\
\text { (lake), PE, 0.3-1 mm } \\
\text { (lake) } 1-2 \text { (shoreline) }\end{array}$ & $\begin{array}{l}\text { Egessa et al. } \\
\text { (2020) }\end{array}$ \\
\hline & 16 & $\begin{array}{l}\text { Donghu Lake, Wuhan, china } \\
\text { Benthic sediment }\end{array}$ & Piston gravity sampler (depth of $57 \mathrm{~cm}$ ) & Fibers, PET, $<0.5 \mathrm{~mm}$ & $\begin{array}{l}\text { Dong et al. } \\
(2020)\end{array}$ \\
\hline & 17 & $\begin{array}{l}\text { Lake Ziway, Ethiopia } \\
\text { Benthic sediment }\end{array}$ & Ekman grab sampler (depth of $0-2 \mathrm{~cm}$ ) & Fragments, PE, 0.15-5 mm & $\begin{array}{l}\text { Merga et al. } \\
(2020)\end{array}$ \\
\hline & 18 & $\begin{array}{l}\text { Donting Lake, China } \\
\text { Littoral sediment and Benthic } \\
\text { sediment }\end{array}$ & $\begin{array}{l}\text { Stainless steel shovel }\left(0.25 \mathrm{~m}^{2} \text { area, }\right. \\
\text { depth of } 2 \mathrm{~cm}) \text {, grab sampler }\end{array}$ & $\begin{array}{l}\text { Fibers, PET, } \\
\text { PE, }<0.5 \mathrm{~mm}\end{array}$ & $\begin{array}{l}\text { Yin et al. } \\
\text { (2020) }\end{array}$ \\
\hline \multirow[t]{6}{*}{$\begin{array}{l}\text { River- } \\
\text { estuary- } \\
\text { lake }\end{array}$} & 1 & $\begin{array}{l}\text { Vembanad Lake, Kerala, } \\
\text { India }\end{array}$ & Van Veen grab $\left(25 \mathrm{~cm}^{2}\right.$ area $)$ & Films, Foams, LDPE & $\begin{array}{l}\text { Sruthy and } \\
\text { Ramasamy } \\
(2017)\end{array}$ \\
\hline & & Benthic sediment & & & \\
\hline & 2 & $\begin{array}{l}\text { Urban water areas } \\
\text { inChangsha, China } \\
\text { Littoral sediment }\end{array}$ & Shovel (depth of $5 \mathrm{~cm}$ ) & Fragments, PS, $<0.5 \mathrm{~mm}$ & $\begin{array}{l}\text { Wen et al. } \\
\text { (2018) }\end{array}$ \\
\hline & 3 & $\begin{array}{l}\text { Coastal plain river network } \\
\text { (Wen-Rui Tang River } \\
\text { watershed) in eastern China } \\
\text { Benthic sediment }\end{array}$ & $\begin{array}{l}\text { Peterson grab }(32 \mathrm{~cm} \times 20 \mathrm{~cm} \text {, depth } \\
\quad \text { of } 0-15 \mathrm{~cm})\end{array}$ & Figments, PE, 20-100 $\mu \mathrm{m}$ & $\begin{array}{l}\text { Z.F.Wang } \\
\text { et al. } \\
\text { (2018) }\end{array}$ \\
\hline & 4 & $\begin{array}{l}\text { Skudai and Tebrau river, } \\
\text { Malaysia } \\
\text { Benthic sediment }\end{array}$ & Box corer & $1001-5000 \mu \mathrm{m}$ & $\begin{array}{l}\text { Sarijan et al. } \\
\text { (2018) }\end{array}$ \\
\hline & 5 & $\begin{array}{l}\text { Cecina river estuary, } \\
\text { Tuscany, Italy } \\
\text { Littoral sediment andBenthic } \\
\text { sediment }\end{array}$ & $\begin{array}{l}\text { Wide mouth glass jars } 1 \mathrm{~L} \text { by scientific } \\
\text { scuba divers (depth of } 5 \mathrm{~cm} \text { ) }\end{array}$ & Fragments, $>500 \mu \mathrm{m}$ & $\begin{array}{l}\text { Blašković } \\
\text { et al. } \\
\text { (2018) }\end{array}$ \\
\hline \multirow[t]{2}{*}{ Lagoon } & 1 & $\begin{array}{l}\text { Lagoon of Venice, Italy } \\
\text { Benthic sediment }\end{array}$ & Box corer (depth of $0-5 \mathrm{~cm}$ ) & Fragments, PE, $<100 \mu \mathrm{m}$ & $\begin{array}{l}\text { Vianello } \\
\text { et al. } \\
\text { (2013) }\end{array}$ \\
\hline & 2 & $\begin{array}{l}\text { Complex Lagoon-Channel of } \\
\text { Bizerte, Northern Tunisia } \\
\text { Littoral sediment }\end{array}$ & $\begin{array}{l}\text { Stainless steel spatula } \\
(0.25 \mathrm{~m} \times 0.25 \mathrm{~m} \text { quadrats, depth of } \\
2-3 \mathrm{~cm})\end{array}$ & Fibers & $\begin{array}{l}\text { Abidli et al. } \\
\text { (2016) }\end{array}$ \\
\hline
\end{tabular}


Table 3 Microplastic studies in water and sediment of inland water bodies around the world

\begin{tabular}{|c|c|c|c|c|c|}
\hline $\begin{array}{l}\text { Study } \\
\text { compartment }\end{array}$ & Number & Study area & Sampling tools & $\begin{array}{l}\text { Dominant microplastic } \\
\text { characteristics (shape, polymer } \\
\text { type, size) }\end{array}$ & Reference \\
\hline \multirow[t]{15}{*}{$\begin{array}{l}\text { River- } \\
\text { estuary }\end{array}$} & 1 & $\begin{array}{l}\text { Three GorgesReservoir, } \\
\text { China }\end{array}$ & $\begin{array}{l}12 \mathrm{~V} \text { DC Teflon pump }(25 \mathrm{~L}), \\
\text { passed through } 48-\mu \mathrm{m} \text { stainless } \\
\text { steel sieve (water) and Van Veen } \\
\text { grab }\left(0.25 \mathrm{~m}^{2} \text { area) (sediment) }\right.\end{array}$ & Fibers, PS, $<0.5 \mathrm{~mm}$ & $\begin{array}{l}\text { Di and } \\
\text { Wang } \\
(2018)\end{array}$ \\
\hline & 2 & $\begin{array}{l}\text { Five urban estuaries of } \\
\text { KwaZulu-Natal, South } \\
\text { Africa }\end{array}$ & $\begin{array}{l}\text { Conical zooplankton net }(300 \mu \mathrm{m} \\
\text { mesh size })(\text { water }) \text { and sediment } \\
\text { corer }(\text { depth of } 10 \mathrm{~cm})(\text { sediment })\end{array}$ & Fragments & $\begin{array}{l}\text { Naidoo } \\
\text { et al. } \\
(2015)\end{array}$ \\
\hline & 3 & $\begin{array}{l}\text { Pearl River along Guangzhou } \\
\text { City, China }\end{array}$ & $\begin{array}{l}\text { Water sampler }(5 \mathrm{~L}) \text { ( water), Van } \\
\text { Veen grab (depth of } 5 \mathrm{~cm}) \\
\text { (sediment) }\end{array}$ & $\begin{array}{l}\text { Fibers, PP, } 0.02-0.5 \mathrm{~mm} \text {, } \\
0.5-1 \mathrm{~mm}\end{array}$ & $\begin{array}{l}\text { Lin et al. } \\
(2018)\end{array}$ \\
\hline & 4 & Antuã River, Portugal & $\begin{array}{l}\text { Water pump }(55 \mu \mathrm{m} \text { mesh size } \\
\text { (water), Van Veen grab (depth of } \\
12 \mathrm{~cm}) \text { (sediment) }\end{array}$ & Foams, PE, PP & $\begin{array}{l}\text { Rodrigues } \\
\text { et al. } \\
\text { (2018) }\end{array}$ \\
\hline & 5 & Ottawa River, Canada & $\begin{array}{l}\text { Bottle sampling and Manta trawls } \\
(100 \mu \mathrm{m} \text { mesh size) (water), } \\
\text { Ekman bottom grab sampler } \\
\text { (sediment) }\end{array}$ & Fibers & $\begin{array}{l}\text { Vermaire } \\
\text { et al. } \\
(2017)\end{array}$ \\
\hline & 6 & $\begin{array}{l}\text { Charleston Harbor and } \\
\text { Winyah Bay, two } \\
\text { developed estuaries in US }\end{array}$ & $\begin{array}{l}\text { Sea surface microlayer collection } \\
\text { apparatus }(4 \mathrm{~L}) \text { (water) and } \\
\text { stainless steel trowel } \\
(0.25 \mathrm{~m} \times 0.25 \mathrm{~m} \text { transect) } \\
\text { (sediment) }\end{array}$ & Fragments, $150-499 \mu \mathrm{m}$ & $\begin{array}{l}\text { Gray et al. } \\
\text { (2018) }\end{array}$ \\
\hline & 7 & $\begin{array}{l}\text { Slum and industrial area of } \\
\text { Ciwalengke River, } \\
\text { Majalaya, Indonesia }\end{array}$ & $\begin{array}{l}\text { Grab samples with glass container } \\
\text { (1L) (water), Ekman grab } \\
\text { sampler and shovel (sediment) }\end{array}$ & - & $\begin{array}{l}\text { Alam et al. } \\
\text { (2019) }\end{array}$ \\
\hline & 8 & Pearl River catchment, China & $\begin{array}{l}\text { Plankton net }(160 \mu \mathrm{m} \text { mesh size } \\
\text { (water), grasp bucket and gravity } \\
\text { corer (depth of } 54 \mathrm{~cm}) \text { (sediment) }\end{array}$ & Sheets, PP, LDPE, $<0.25 \mathrm{~mm}$ & $\begin{array}{l}\text { Fan et al. } \\
\text { (2019) }\end{array}$ \\
\hline & 9 & Wei River, China & $\begin{array}{l}\text { Bulk sampling using clean pump } \\
\text { (5L) passed through } 75 \mu \mathrm{m} \text { mesh } \\
\text { size (water), grab (sediment) }\end{array}$ & Fibers, $<0.5 \mathrm{~mm}$ & $\begin{array}{l}\text { Ding et al. } \\
\text { (2019) }\end{array}$ \\
\hline & 10 & $\begin{array}{l}\text { Tibet Plateau Rivers, China } \\
\text { (Buqu River (the source of } \\
\text { the Yangtze }\end{array}$ & $\begin{array}{l}\text { Large flow sampler (water) and a } \\
\text { stainless steel shovel (depth of } \\
2 \mathrm{~cm} \text { ) (sediment) }\end{array}$ & $\begin{array}{l}\text { Fibers, PET (sediment samples), } \\
\text { PE (water samples), }<0.5 \mathrm{~mm}\end{array}$ & $\begin{array}{l}\text { Jiang et al. } \\
\text { (2019) }\end{array}$ \\
\hline & 11 & $\begin{array}{l}\text { Middle and lower reaches of } \\
\text { the Yangtze River, China }\end{array}$ & $\begin{array}{l}\text { A fishery administration vessel } \\
\text { (AVANI trawl net } 333 \mu \mathrm{m} \text { mesh } \\
\text { size), A plankton net ( } 64 \mu \mathrm{m} \\
\text { mesh size) (water) and grab } \\
\text { sampler (sediment) }\end{array}$ & $\begin{array}{l}\text { Sheets, PP, } 0.3-0.5 \mathrm{~mm} \text {, } \\
0.5-1 \mathrm{~mm}\end{array}$ & $\begin{array}{l}\text { Xiong } \\
\text { et al. } \\
\text { (2019) }\end{array}$ \\
\hline & 12 & Nakdong River, South Korea & $\begin{array}{l}\text { Stainless steel beaker, submersible } \\
\text { pump (water), Van Veen grab } \\
\text { (depth of } 2 \mathrm{~cm} \text { ) (sediment) }\end{array}$ & Fragments, PP & $\begin{array}{l}\text { Eo et al. } \\
(2019)\end{array}$ \\
\hline & 13 & Mohawk River, USA & $\begin{array}{l}\text { Manta trawl }(333 \mu \mathrm{m} \text { mesh size } \\
\text { (water) and Ekman grab sampler } \\
\text { was (sediment) }\end{array}$ & Fibers, fragments & $\begin{array}{l}\text { Smith } \\
\text { et al. } \\
(2017)\end{array}$ \\
\hline & 14 & $\begin{array}{l}\text { Ebro River Delta, } \\
\text { Northeastern Iberian } \\
\text { Peninsula, Spain }\end{array}$ & $\begin{array}{l}\text { Neuston net }(5 \mu \mathrm{m} \text { mesh size) } \\
\text { (water), stainless steel spoon } \\
(0.2 \mathrm{~m} \times 0.2 \mathrm{~m} \text { quadrant, depth } \\
\text { of } 2.5 \mathrm{~cm}) \text { and van Veen grab } \\
\text { sampler (sediments) }\end{array}$ & Fibers, PE, 200-500 $\mu \mathrm{m}$ & $\begin{array}{l}\text { Simon- } \\
\text { Sánchez } \\
\text { et al. } \\
(2019)\end{array}$ \\
\hline & 15 & $\begin{array}{l}\text { Yongjiang River, Nanning } \\
\text { City, South China }\end{array}$ & $\begin{array}{l}12 \mathrm{~V} \text { DC Teflon pump }(10 \mathrm{~L}) \\
\text { passed through } 50-\mu \mathrm{m}-\mathrm{mesh} \text { size } \\
\text { sieve (water) and Van Veen grab } \\
\text { (sediment) }\end{array}$ & $\begin{array}{l}\text { Fibers, PE, } 330-1000 \mu \mathrm{m} \text { and } \\
1-3 \mathrm{~mm}\end{array}$ & $\begin{array}{l}\text { Zhang } \\
\text { et al. } \\
(2020)\end{array}$ \\
\hline
\end{tabular}


Table 3 (continued)

\begin{tabular}{|c|c|c|c|c|c|}
\hline $\begin{array}{l}\text { Study } \\
\text { compartment }\end{array}$ & Number & Study area & Sampling tools & $\begin{array}{l}\text { Dominant microplastic } \\
\text { characteristics (shape, polymer } \\
\text { type, size) }\end{array}$ & Reference \\
\hline & 16 & Maozhou River, China & $\begin{array}{l}\text { Stainless steel bucket (5L), depth } \\
\text { of } 50 \mathrm{~cm} \text { (water) box corer, depth } \\
\text { of } 20 \mathrm{~cm} \text { (sediment) }\end{array}$ & Fragments, $10 \mu \mathrm{m}-0.1 \mathrm{~mm}$ & $\begin{array}{l}\text { Wu et al. } \\
(2020)\end{array}$ \\
\hline & 17 & $\begin{array}{l}\text { Chao Phraya River, } \\
\text { Bangkok, Thailand }\end{array}$ & $\begin{array}{l}\text { Manta trawl } 2 \mathrm{~m} \text { long, width of } \\
50 \mathrm{~cm} \text {, and a height of } 20 \mathrm{~cm} \text {, } \\
300 \mu \mathrm{m} \text { mesh size (water), Van } \\
\text { Veen grab sampler (sediment) }\end{array}$ & $\begin{array}{l}\text { Fragments, PP, 0.5-1.0 (water } \\
\text { samples) } 0.053-0.5 \mathrm{~mm} \\
\text { (sediment samples) }\end{array}$ & $\begin{array}{r}\text { Ta et al. } \\
(2020)\end{array}$ \\
\hline & 18 & $\begin{array}{l}\text { Ravi River in urban center ( } \\
\text { predominant drains and } \\
\text { canals of Lahore district), } \\
\text { Lahore, Pakistan }\end{array}$ & $\begin{array}{l}\text { Stainless steel spatula, } \\
0.3 \mathrm{~m} \times 0.3 \mathrm{~m} \text { quadrate, depth } \\
\text { of } 1 \mathrm{~cm} \text { (sediment) }\end{array}$ & $\begin{array}{l}\text { Fragments, PE, 150-300 } \mu \mathrm{m} \\
\text { (sediment samples), and large } \\
\text { size MPs } 300 \mu \mathrm{m}-5 \mathrm{~mm} \text { (water } \\
\text { samples) }\end{array}$ & $\begin{array}{l}\text { Irfan et al. } \\
\quad(2020)\end{array}$ \\
\hline & 19 & Magdalena River, Colombia & $\begin{array}{l}\text { Neuston net } 20 \mu \mathrm{m} \text { mesh size } \\
\text { (water), metal shovel, depth of } \\
5 \mathrm{~cm} \text { (sediment) }\end{array}$ & Fibers, PP & $\begin{array}{l}\text { Martínez } \\
\text { Silva } \\
\text { and } \\
\text { Nanny } \\
\text { (2020) }\end{array}$ \\
\hline \multirow[t]{5}{*}{$\begin{array}{l}\text { Lake- } \\
\text { reservoir }\end{array}$} & 1 & $\begin{array}{l}\text { Lake Bolsena and Lake } \\
\text { Chiusi, Italy }\end{array}$ & $\begin{array}{l}\text { Manta trawl } 300 \mu \mathrm{m} \text { mesh size } \\
60 \mathrm{~cm} \times 18.5 \mathrm{~cm} \text { (water), } 0.25 \\
\mathrm{~m}^{2} \text { area, depth of } 3 \mathrm{~cm} \\
\text { (sediment) }\end{array}$ & Fibers, $<0.3 \mathrm{~mm}, 0.3-0.5 \mathrm{~mm}$ & $\begin{array}{l}\text { Fischer } \\
\text { et al. } \\
(2016)\end{array}$ \\
\hline & 2 & Dongting Lake, China & $\begin{array}{l}\text { Flow sampler (30L), passed } \\
\text { through } 45 \mu \mathrm{m} \text { mesh size (water) } \\
\text { and Stainless shovel } \\
0.3 \mathrm{~m} \times 0 . .2 \mathrm{~m} \text { quadrat, depth of } \\
0-2 \mathrm{~cm} \text { (sediment) }\end{array}$ & $\begin{array}{l}\text { Fibers, PET (sediment sediment) } \\
\text { PE (water sediment) },<0.5 \mathrm{~mm}\end{array}$ & $\begin{array}{l}\text { Jiang et al. } \\
\text { (2018) }\end{array}$ \\
\hline & 3 & Six dams near Ithaca, USA & $\begin{array}{l}\text { Grab sample } 1 \mathrm{~L} \text { plastic bottles } \\
\text { (water), plastic scoop (sediment } \\
\text { samples) }\end{array}$ & Fibers & $\begin{array}{l}\text { Watkins } \\
\text { et al. } \\
\text { (2019a) }\end{array}$ \\
\hline & 4 & $\begin{array}{l}\text { Danjiangkou Reservoir, } \\
\text { China }\end{array}$ & $\begin{array}{l}12 \text { V DC Teflon pump (20L), depth } \\
\text { of } 0-20 \mathrm{~cm} \text { (water), grab } \\
\text { (sediment) }\end{array}$ & $\begin{array}{l}\text { Fibers, PP, group } 1 \\
\quad(48 \mu \mathrm{m}-0.5 \mathrm{~mm}) \text {, group } 2 \\
\quad(0.5-1 \mathrm{~mm}), \text { group } 3(1-2 \mathrm{~mm}) \text {, }\end{array}$ & $\begin{array}{r}\text { Di et al. } \\
(2019)\end{array}$ \\
\hline & 5 & $\begin{array}{l}\text { Lakes along the middle and } \\
\text { lower reaches of Yangtze } \\
\text { River Basin, China }\end{array}$ & $\begin{array}{l}\text { Neuston plankton net } 74 \mu \mathrm{m} \text { mesh } \\
\text { size (water), Van Veen grab } \\
\text { (sediment) }\end{array}$ & Fibers, PET, 20-50 $\mu \mathrm{m}$ & $\begin{array}{l}\text { Li et al. } \\
(2019)\end{array}$ \\
\hline Stram & 1 & $\begin{array}{l}18 \text { streams in and around the } \\
\text { city of Auckland, New } \\
\text { Zealand }\end{array}$ & $\begin{array}{l}\text { Phytoplankton net } 63 \mu \mathrm{m} \text { mesh } \\
\text { size (water) and scooped with } \\
\text { container, depth of } 5 \mathrm{~cm} \\
\text { (sediment) }\end{array}$ & $\begin{array}{l}\text { Fragments, fibers (water samples), } \\
\text { fragments (sediment samples), } \\
\text { poly(hexadecyl) methacrylate } \\
\text { (PHM), ethylene/ethyl acrylate } \\
\text { copolymer (EEAC), 63-500 } \mu \mathrm{m}\end{array}$ & $\begin{array}{l}\text { Dikareva } \\
\text { and } \\
\text { Simon } \\
\text { (2019) }\end{array}$ \\
\hline Fish ponds & 1 & $\begin{array}{l}\text { Central and Eastern } \\
\text { European region }\end{array}$ & $\begin{array}{l}\text { Jet pumps, passed through } 2 \mathrm{~mm} \\
\text { mesh size strainer, depth of } \\
10-20 \mathrm{~cm} \text { (water), Veen grab } \\
\text { sampler and a hand spade } \\
\text { (sediment samples) }\end{array}$ & $\mathrm{PP}$ & $\begin{array}{l}\text { Bordós } \\
\text { et al. } \\
\text { (2019 }\end{array}$ \\
\hline $\begin{array}{l}\text { River- } \\
\text { estuary- } \\
\text { lake- } \\
\text { WWTPs }\end{array}$ & 1 & $\begin{array}{l}\text { River Barrow, River, Nore, } \\
\text { Lough, Lurgan (Cushina, } \\
\text { Co. Offaly) and River } \\
\text { Liffey (Newbridge, Co. } \\
\text { Kildare), Ireland }\end{array}$ & - & PS & $\begin{array}{l}\text { Cedro and } \\
\text { Cleary } \\
2015\end{array}$ \\
\hline
\end{tabular}


Table 4 Microplastic studies in water, sediment and biota of inland water bodies around the world

\begin{tabular}{|c|c|c|c|c|c|}
\hline $\begin{array}{l}\text { Water } \\
\text { compartment }\end{array}$ & Number & Study area & Sampling tools & $\begin{array}{l}\text { Dominant } \\
\text { microplastic } \\
\text { characteristics } \\
\text { (shape, polymer } \\
\text { type, size) }\end{array}$ & Reference \\
\hline \multirow[t]{5}{*}{$\begin{array}{l}\text { River- } \\
\text { estuary- } \\
\text { lake }\end{array}$} & 1 & $\begin{array}{l}\text { Xiangxi Bay of Three Gorges } \\
\text { Reservoir, China }\end{array}$ & $\begin{array}{l}\text { Surface trawl } 50 \times 100 \times 150 \mathrm{~cm}, \\
112 \mu \mathrm{m} \text { mesh size (water) and Petersen } \\
\text { grab (sediment) }\end{array}$ & $\begin{array}{l}\text { Sheets, PP, } \\
1-5 \mathrm{~mm}\end{array}$ & $\begin{array}{l}\text { Zhang } \\
\text { et al. } \\
(2017\end{array}$ \\
\hline & 2 & Qinghai Lake, China & $\begin{array}{l}\text { Trawl net } 50 \times 100 \times 150 \mathrm{~cm}, 112 \mu \mathrm{m} \\
\text { mesh size (water) and stainless steel } \\
\text { shovel, } 0.2 \mathrm{~m} \times 0.2 \mathrm{~m} \text { quadrate, depth } \\
\text { of } 0-2 \mathrm{~cm} \text { (sediment) }\end{array}$ & $\begin{array}{l}\text { Sheets, PP,PE, } \\
0.112-0.5 \mathrm{~mm}\end{array}$ & $\begin{array}{l}\text { Xiong } \\
\text { et al. } \\
(2018\end{array}$ \\
\hline & 3 & Taihu Lake, China & $\begin{array}{l}\text { Nylon plankton net } 333 \mu \mathrm{m} \text { mesh size } \\
\text { and steel sampler ( } 5 \mathrm{~L}) \text { (water), Peterson } \\
\text { sampler (sediment) and a bottom fauna } \\
\text { trawl (biota) }\end{array}$ & $\begin{array}{l}\text { Fibers, } \\
100-333 \mu \mathrm{m}, \\
333-1000 \mu \mathrm{m}\end{array}$ & $\begin{array}{l}\text { Su et al., } \\
2016\end{array}$ \\
\hline & 4 & Lake Geneva, Switzerland & $\begin{array}{l}\text { Manta trawl } 300 \mu \mathrm{m} \text { mesh size (water), } \\
\text { fishes and birds were collected by a } \\
\text { fisherman }\end{array}$ & $<5 \mathrm{~mm}, \mathrm{PS}$ & $\begin{array}{l}\text { Faure } \\
\text { et al. } \\
(2012\end{array}$ \\
\hline & 5 & Poyang Lake, China & $\begin{array}{l}\text { Steel sampler }(20 \mathrm{~L}) \text {, passed through } \\
50 \mu \mathrm{m} \text { mesh size (water), Van Veen } \\
\text { grab (sediment), fish samples were } \\
\text { obtained from an aquatic product } \\
\text { market }\end{array}$ & $\begin{array}{l}\text { Fibers, PP, } \\
0.1-0.5 \mathrm{~mm}\end{array}$ & $\begin{array}{l}\text { Yuan } \\
\text { et al. } \\
\text { (2019 }\end{array}$ \\
\hline \multirow[t]{2}{*}{$\begin{array}{l}\text { River- } \\
\text { estuary- } \\
\text { lake }\end{array}$} & 1 & $\begin{array}{l}\text { Six of the largest Swiss lakes and some } \\
\text { rivers-Switzerland }\end{array}$ & $\begin{array}{l}\text { Manta trawl } 300 \mu \mathrm{m} \text { mesh size (water), } \\
\text { multi-mesh gillnets and vertical benthic } \\
\text { and pelagic nets (biota) }\end{array}$ & $\begin{array}{l}\text { Fragments, } \\
\quad \mathrm{PE},>300 \mu \mathrm{m}\end{array}$ & $\begin{array}{l}\text { Faure } \\
\text { et al. } \\
(2015\end{array}$ \\
\hline & 2 & $\begin{array}{l}\text { Middle-Lower Yangtze River Basin, } \\
\text { China }\end{array}$ & $\begin{array}{l}\text { Steel bucket }(5 \mathrm{~L}) \text {, depth of } 0.12 \mathrm{~cm} \\
\text { (water), Peterson sampler, depth of } \\
10 \mathrm{~cm} \text { (sediment), bottom fauna trawls } \\
\text { (biota) }\end{array}$ & $\begin{array}{l}\text { Fibers, PES, } \\
0.25-1 \mathrm{~mm}\end{array}$ & $\begin{array}{l}\text { Su et al. } \\
\quad(2018\end{array}$ \\
\hline Pond & 1 & Storm water pond, Viborg, Denmark & $\begin{array}{l}\text { Bulk samples }(10 \mathrm{~L}) \text {, glass bottles, depth } \\
\text { of } 10 \mathrm{~cm} \text { (water), Sediment corer, depth } \\
\text { of } 5-8 \mathrm{~cm} \text { (sediment), gill net and } \\
\text { fishing net (biota) }\end{array}$ & $\mathrm{PP}$ & $\begin{array}{l}\text { Olesen } \\
\text { et al. } \\
(2019\end{array}$ \\
\hline $\begin{array}{l}\text { Small water } \\
\text { bodies }\end{array}$ & 1 & YangtzeRiver Delta, China & $\begin{array}{l}\text { Steel bucket (water), stainless steel } \\
\text { spatula, depth of } 0-5 \mathrm{~cm} \text { (sediment) }\end{array}$ & $\begin{array}{l}\text { Fibers, } \\
\text { PES, }<0.5 \mathrm{~mm}\end{array}$ & $\begin{array}{l}\text { Hu et al. } \\
\quad(2018\end{array}$ \\
\hline $\begin{array}{l}\text { River, } \\
\text { Canal, } \\
\text { WWTPs, } \\
\text { Sea }\end{array}$ & 1 & $\begin{array}{l}\text { Dutch river delta and Amsterdam } \\
\text { canals, wastewater treatment plants, } \\
\text { North Sea sediments and biota, } \\
\text { Netherland and Germany }\end{array}$ & $\begin{array}{l}\text { Bulk sample, glass bottles (2L) (water) } \\
\text { and grab samples and Van Veen grab } \\
\text { (sediment) }\end{array}$ & Fibers, $10-300 \mu \mathrm{m}$ & $\begin{array}{l}\text { Leslie } \\
\text { et al. } \\
(2017\end{array}$ \\
\hline
\end{tabular}

\section{Pump samplers and grab samples}

Volume reduction pump sampler and grab samples are also used in some of the research papers. Pump sampling consist of pumping water manually or using a motor through an inline filter. Grab sampling method includes using a bucket to collect water and sieve the water in the field (Han et al. 2020; Miller et al. 2017; Y. Mao et al. 2020a). A fixed amount of bottle is also submerged, filling with surface water for laboratory analysis (Barrows et al. 2018; Dubaish and Liebezeit 2013). Water collected using pumps or bulk samplers is taken from different depths with different volumes. Due to the high variability of microplastic spatial distribution, the sampling area covered is limited and using a pump or bulk sampler may not be representative. Therefore, taking multiple replicates is suggested (Zhang et al. 2018). However, pumps can be used to collect large volumes of water, which may be advantageous in areas where the density of microplastics is suspected to be low (Crawford and Quinn 2017). Water volume could be variable from $5 \mathrm{~mL}$ to $500 \mathrm{~L}$ (Braun et al. 2018). In addition, they do not possess the limitation caused by pacific sampling mesh tool. Based on literature reviews, significantly more microplastic particles are present in smaller size ranges. A combination of volume-reduced netbased sampling and bulk sampling seems to be very helpful 
Fig. 2 Frequency and trend of studies $(n=150)$ on the presence of microplastic particles in freshwater environment in different matrix including water, sediment, water + sediment, water or sediment + biota and water + sediment + biota

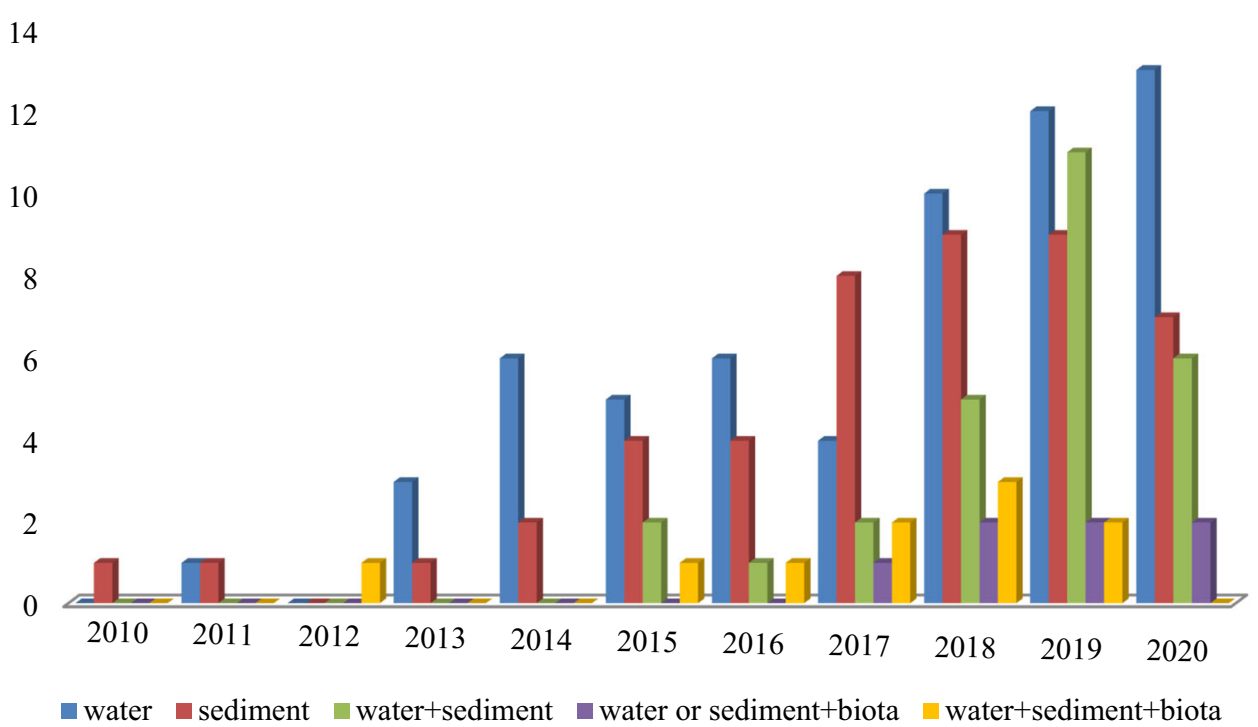

in estimating the missing fractions and enables a greater spatial resolution (Fischer et al. 2016).

\section{Comparison between different water sampling methods}

In the comparison of manta trawling and pump sampling methods in microplastic sampling from water of Lake Tollense, Germany, different results were observed in the abundance of microplastics, microplastics shape and size. It was suggested that manta trawl is not sufficient in retaining fibers and small microplastics from water samples. Therefore, the pump sampling approach with the filtration of large water volumes is necessary to generate reliable results. However, the pump sampling covers small microplastics. Small plastic particles are greater in number. Volume-reduced sampling covers large microplastics, being less abundant but still important. Fibers detected in the manta samples were unevenly spread across the whole size range. Fibers found in the pump samples showed distinct positively skewed distribution peaking at $>$ $500-600 \mu \mathrm{m}$ in length. The most abundant polymer composition in manta trawl samples was polyethylene and polyethylene terephthalate for the pump sampling method (Tamminga et al. 2020).

Lahens and colleagues utilized a bucket and $300-\mu \mathrm{m}$ plankton net. Bulk water sampling was used for anthropogenic fiber analysis and 300- $\mu \mathrm{m}$-mesh size plankton net exposition for fragment analysis (Lahens et al. 2018). In the study of $\mathrm{Su}$ and colleagues, the average abundance of microplastics was found to be higher in plankton net samples rather than bulk surface water samples ( $\mathrm{Su}$ et al. 2016). In general, water sampling volumes depend on the solid richness and the target microplastic size range. Barrows et al. (2017) compared grab samples to the conventional neuston net approach. Grab samples collected three orders of magnitude more microplastics than the net approach (Barrows et al. 2017).

In comparison between manta trawl and in situ pump filtration methods, it was found that the pump sampling method is more accurate in volume measurement and versatile for point sampling and filter size choice. However, due to the lower sampling volume, it might be more suitable for sampling in areas with a higher level of contamination. On the other hand, the trawling method has the ability to cover and sample a larger area and therefore overcomes some of the problems related to patchiness (Karlsson et al. 2020). A combination of volume-reduced net-based sampling and bulk sampling seems to be very effective in comprehensive monitoring of microplastic in aquatic environment.

\section{Sediment compartment}

Because of such characteristics as buoyancy and extreme durability, synthetic polymers are present in rivers, lakes and oceans and accumulate in sediments all over the world. Microplastic durability makes it highly resistant to degradation from decades to millennia in its polymer forms (Mathalon and Hill 2014). Small plastic particles are easily accessible to a wide range of aquatic organisms, accumulating in their cells and tissues and ultimately transferred through the food web. Most plastics are extremely durable and persistent (Sharma and Chatterjee 2017).

Microplastics in water compartment may be diluted due to seasonal variation in water volume and water dynamic behavior. For sediment compartments, with a static environment, dilution barely happens, and sediments can easily act as accumulation environments. Sediments are a site of 
microplastics accumulation and the habitat of benthic organisms, which are key components of food webs. In microplastic scientific assessment, sediment samples are taken from both the subtidal and benthic part of freshwater bodies. This issue affects the life quality of the organisms in ecosystems, both in benthic and littoral sediment zone. For example, microplastics were recorded in fecal samples and feathers of waterbirds from contaminated wetlands in South Africa. Plastic particles can fill the gizzard and possibly block the pyloric valve leading into the intestine (Reynolds and Ryan 2018). Microplastics were present in the benthic fish species and benthic organisms of the Caspian Sea, and the abundance of plastic particles in animals near the shore was greater than in the central part (Bagheri et al. 2020). High doses of microplastics led to fewer species and fewer juvenile isopods and periwinkles in European flat oysters and their associated benthic communities (Green 2016).

Sampling tools are selected with regard to sampling places. Sampling is performed in various directives with respect to the analysis of nutrients or pollutants, such as metal ions or persistent organic substances (Braun et al. 2018).

\section{Manual grab samplers}

Sediment manual grab methods utilize tools such as hand spades and stainless steel spoon for littoral and beach environments (Fig. 3). As sampling of sediments is facilitated compared to that of the water column, monitoring shore sediments appears to be advantageous. Moreover, non-buoyant particles can be analyzed in sediment samples rather than in water surface samples (Klein et al. 2015). Therefore, sampling from different compartments can give a comprehensive outlook of microplastic pollution problem.

\section{Deep sediment samplers}

Different kinds of grabs and corers are suggested to be suitable for deeper sediment sampling. Ekman and Van Veen grab samplers are deployed to study benthic sediment (Merga et al. 2020; Neto et al. 2019; Sruthy and Ramasamy 2017). Deep sediment sampler can provide a look at the changing abundance and microplastic debris in lake sediments that span a century to present day. These particle can contribute to the discussion on plastic wastes as stratigraphic markers for the Anthropocene (Turner et al. 2019; Vaughan et al. 2017). For specimen transition, the use of glass bottle is recommended. However, plastic or aluminum foil bags can also be used. In the case of plastic container utilization, blank control should be included to prevent bias in the study results (Zhang et al. 2018). Care must also be taken to homogenize the sample during further processing.

\section{Comparison between different sediment sampling methods}

Surface sediment in shore zone could reflect long-term interfacial interaction between waters and terrestrial environment (Yu et al. 2016). Shore sediment sampling consists of multiple transects at a right angle from the water line and the placement of quadrats along the transects. Transects may be visually scanned for bigger microplastics in the field. The surface layer can be removed to a proximate depth and sieved or transferred to the laboratory for separation steps (Ballent et al. 2016; Egessa et al. 2020). Variation in plastic abundance at different natural beach zones (water line, drift line and high-water line) in Lake Garda was investigated. Results showed that the water line contained the lowest level of plastic particles, whereas the highest proportion of plastic debris was observed in the drift line and high-water line (Imhof et al. 2018). Core samples have the advantage of being able to see depth profile and to study potential microplastic trends in considerable time periods. Surface analysis of these microplastics may show higher degradation effects due to longer time period. However, surface particles may be more exposed to degradation factors.

Sediment sampling tools are selected with regard to sampling places and sampling purposes, as they may show different aspects.

\section{Sample preservation}

Samples were are usually preserved with 5\% methyl aldehyde and stored at $4{ }^{\circ} \mathrm{C}$ before analysis (Zhang et al. 2015) or fixed in $2.5 \%$ formalin (Zhao et al. 2014) or submerged in $\sim 40 \%$ ethanol (EtOH) (Mani and Burkhardt-Holm 2020).

\section{Discussion}

No standardized methods exist for selecting mesh size, sampling, clean up, enrichment and detection, making the comparison of different studies complicated. Improving methods is needed to save time and effort in identifying microplastics in different compartments. To the best of our knowledge, water compartments are the most investigated matrix, assessed for microplastics in freshwater 
Fig. 3 Microplastics sampling tools in freshwater studies; a manta trawl, b plankton net, c Petite ponar grab d Van Veen garb, e Ekman grab sampler, f box corer, $\mathbf{g}$ sediment corer, $\mathbf{h}$ metal pail, $\mathbf{i}$ showel, $\mathbf{j}$ trowel
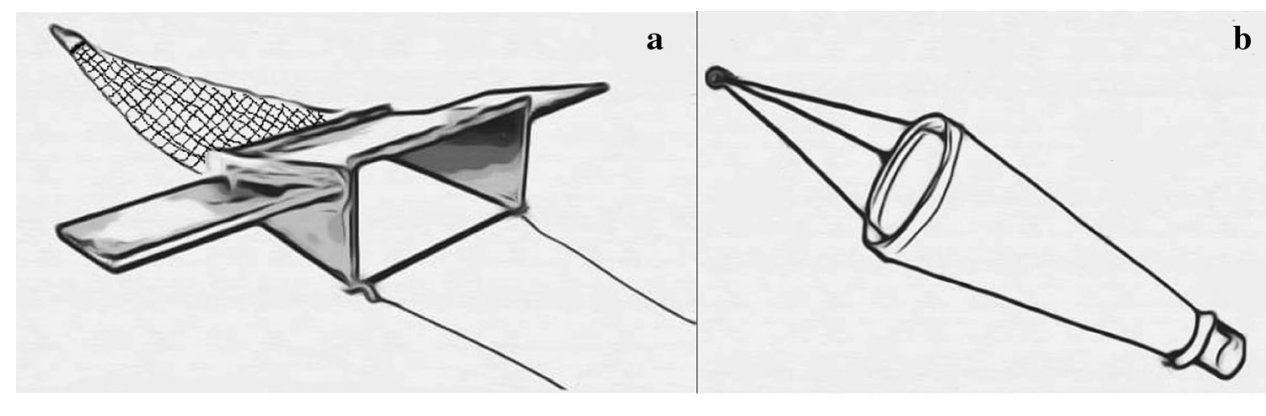

c

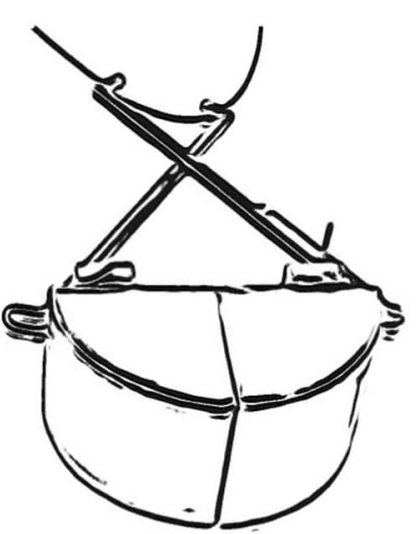

d

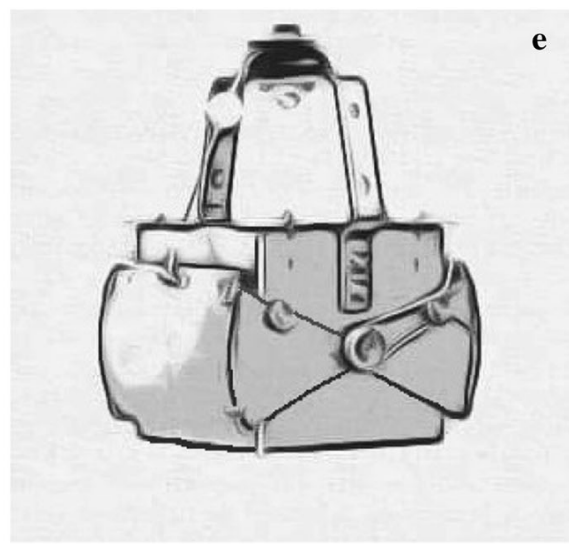

e

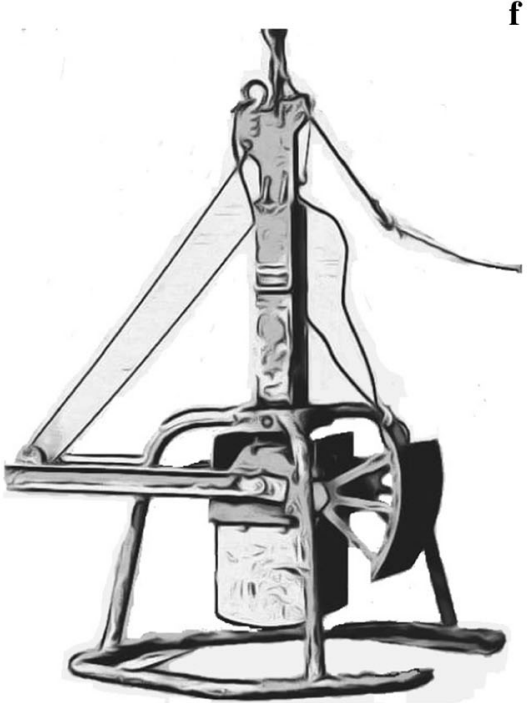

environments (102/150). Rivers and estuarine systems are the most frequently studied compartments for microplastic detection, both in water and in sediment (85/150). This may be due to the reported importance of rivers and estuaries as a vector for microplastics transfer to seas and oceans. Littoral or shore sediments and bottom sediment are frequently assessed for microplastics in reviewed studies. Many microplastic studies used Manta nets to collect surface water samples (Fig. 4). Van Veen grabs and simple hand tools like trowels and stainless steel spoons were the most frequently used tools for the bottom and littoral sediments, respectively (Fig. 5). They are also the most common sediment sampling tools from benthic and beach zones and seem to be appropriate for microplastic studies. Regarding sampling tool characteristics, both false positive and false negative results in analyses of small microplastics occur. In recent studies, there is a tendency to detect microplastics in both water and sediment at the same time. The simultaneous detection of microplastics in the water and sediment compartment gives a better perspective 
Fig. 3 continued

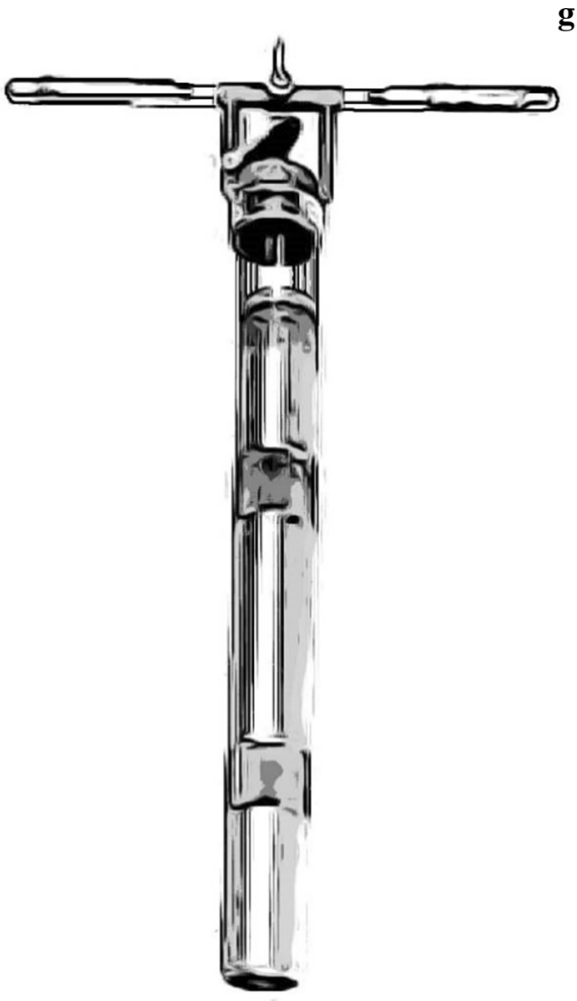

g
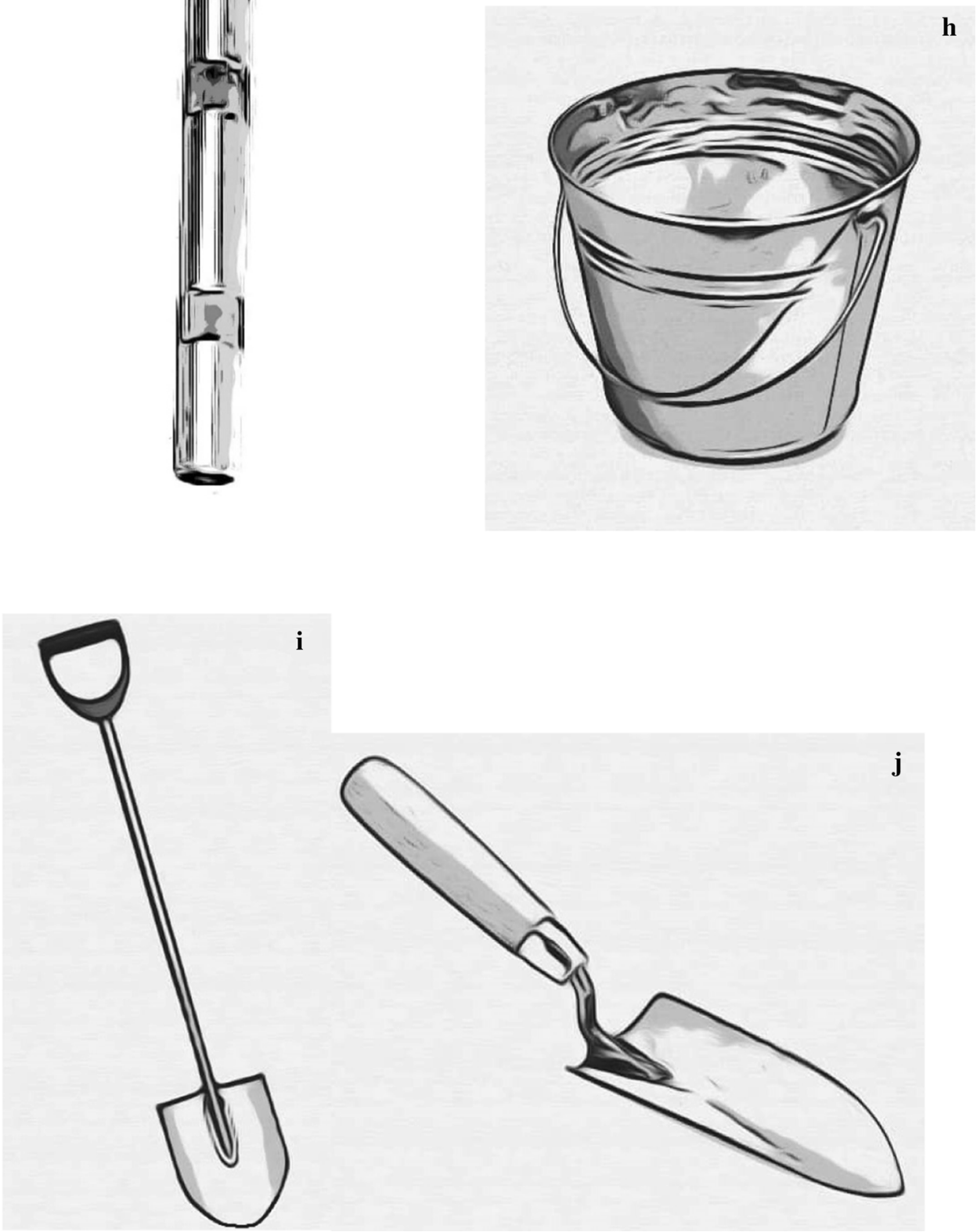

of the situation in the ecosystem. The lack of uniformity in reporting the numbers of microplastics, mostly due to employing different units, is considerably noticeable in reviewing papers. This makes the comparison of results difficult and challenging. Some studies have reported microplastic numbers or weights per volume of sampled water or per total dry matter for sampled sediments (particles $/ \mathrm{kg}$ ); the latter is highly recommended. 
Fig. 4 Frequency of water sampling tools, used in microplastic freshwater studies

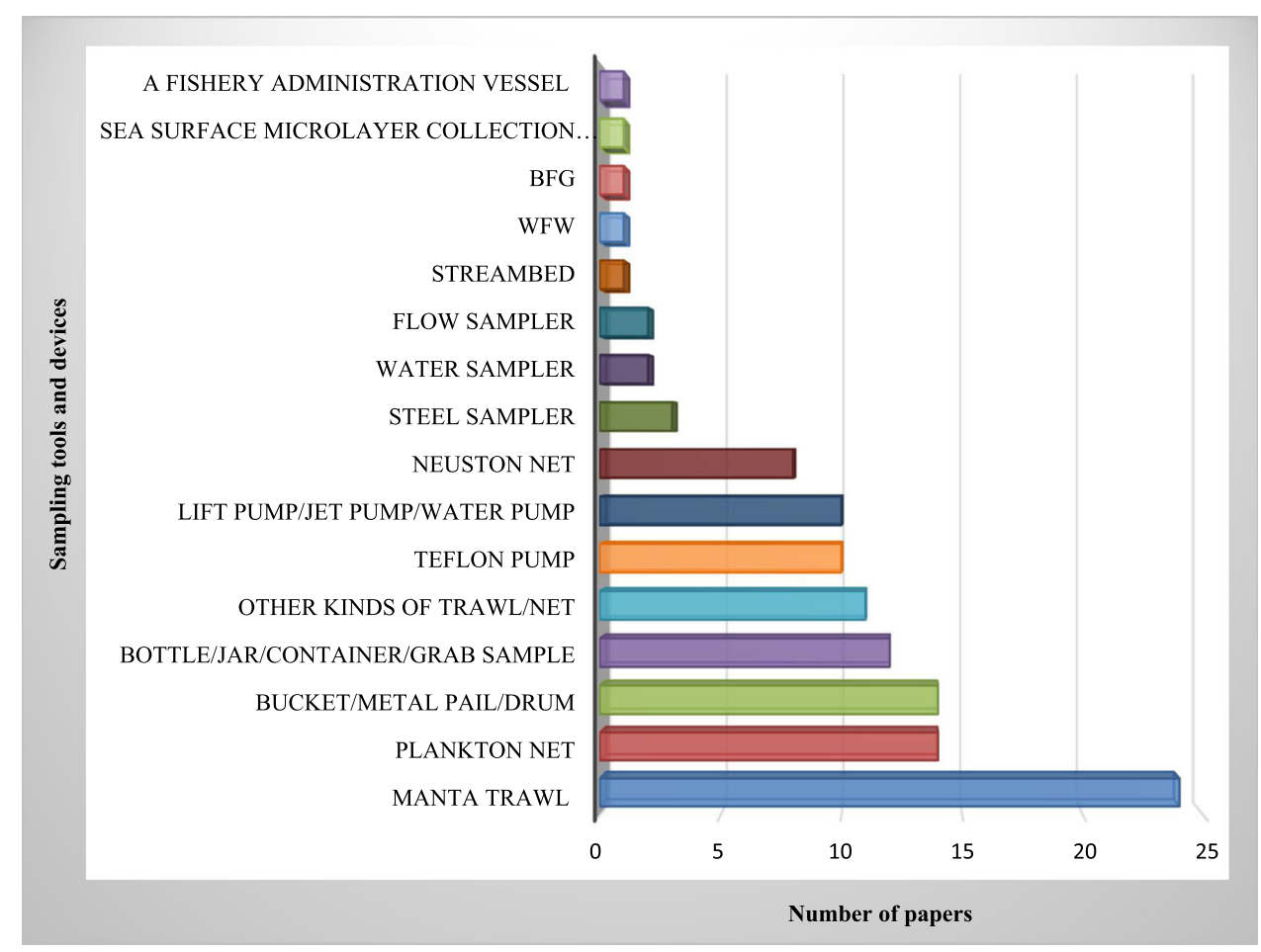

Fig. 5 Frequency of sediment sampling tools, used in microplastic freshwater studies

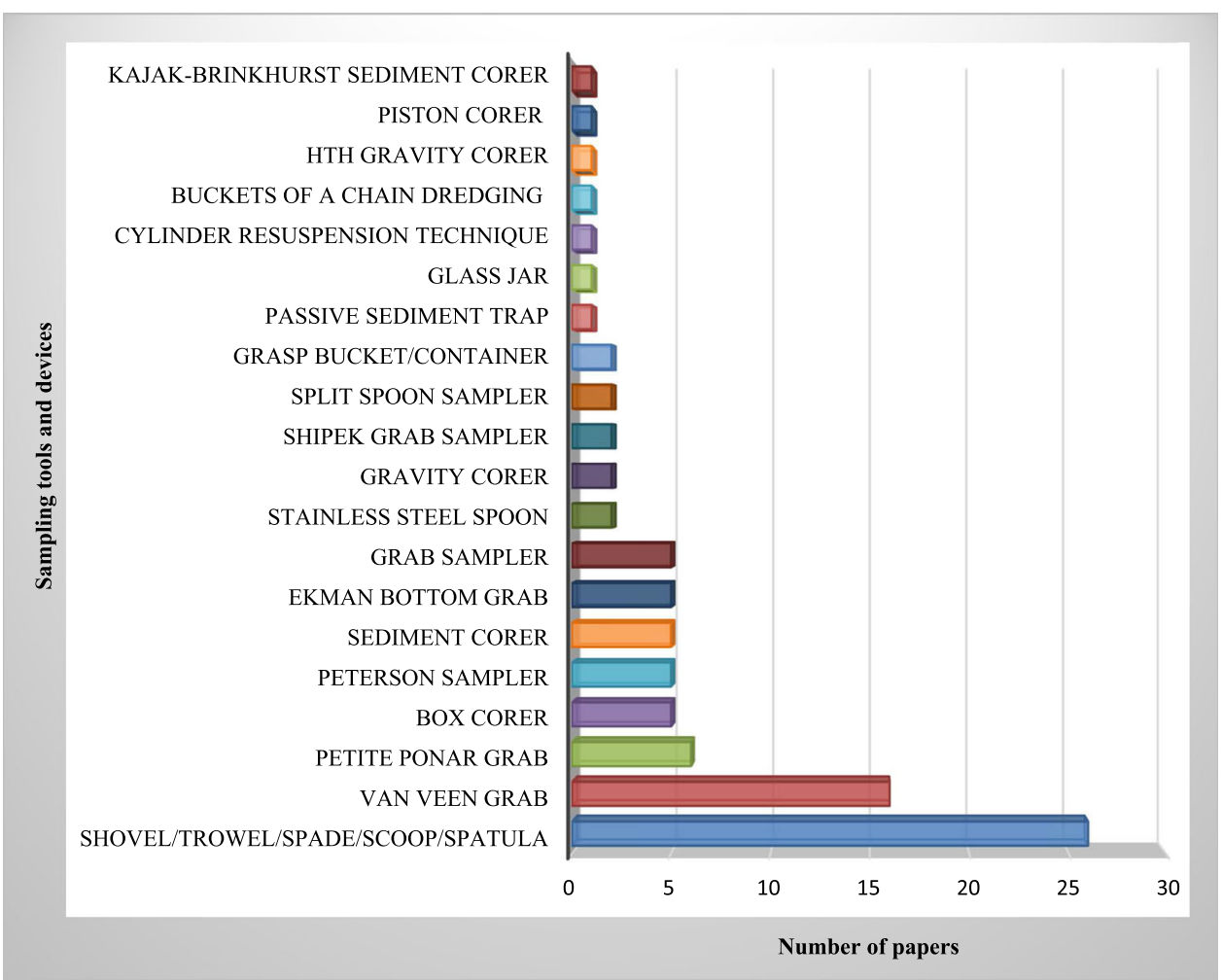

\section{Conclusion}

Employing suitable and reliable sampling, treatment and identification methods is crucial to evaluate microplastic pollution. Sampling and experimental techniques should be standardized to more effectively assess microplastics. Although a smaller mesh size is more appropriate, the choice of trawl or sieve mesh size depends greatly on the study purpose. The kind of environment being studied, e.g., a dynamic river with high water velocity or a calm 
eutrophic lake or wetland, is also of importance. The exact sampling volume, place and depth must be chosen carefully to ensure that samples represent water body characteristics. Sample volumes should be large enough to minimize overestimation induced by scaling up results, especially for water samples. The pump sampling approach with the filtration of large water volumes is necessary to generate reliable results in the spatial association between microplastic pollution in the surface waters and sediments. The trawling method has the ability to cover a larger area during sampling. To cover different microplastic size and shape, it is advantageous to combine both volume reduction and bulk sampling methods for surface water. More research is required to extend the understanding of representative in the study of microplastics as a key factor for the potential development of reliable data.

Authors' contributions Razeghi and Hamidian had the idea for the article, Razeghi performed the literature search and data analysis, Razeghi and Hamidian drafted, and $\mathrm{Wu}$, Zhang and Yang critically revised the work.

Funding This manuscript was supported by Iran National Science Foundation (INSF) under the contract No. 97002416 and CHINESE ACADEMY OF SCI CAS PRESIDENT'S INTERNATIONAL FELLOWSHIP INITIATIVE Grant No. 2021VEA0004.

\section{Declarations}

Conflicts of interest The authors have no conflict of interests to disclose.

Consent for publication All authors agreed with the content and all gave explicit consent to submit, and they obtained consent from the responsible authorities.

\section{References}

Abbasi S, Turner A (2021) Human exposure to microplastics: a study in Iran. J Hazard Mater 403:123799. https://doi.org/10.1016/j. jhazmat.2020.123799

Abidli S, Toumi H, Lahbib Y, El Menif NT (2017) The first evaluation of microplastics in sediments from the complex lagoon-channel of Bizerte (Northern Tunisia). Water Air Soil Pollut 228(7):262. https://doi.org/10.1007/s11270-017-3439-9

Alam FC, Sembiring E, Suendo MBS, V, (2019) Microplastic distribution in surface water and sediment river around slum and industrial area (case study: Ciwalengke River, Majalaya district, Indonesia). Chemosphere 224:637-645. https://doi.org/10.1016/ j.chemosphere.2019.02.188

Alavian SS, Hamidian AH, Ashrafi S, Eagderi S, Khazaei M (2018) Study on age-related bioaccumulation of some heavy metals in the soft tissue of rock oyster (Saccostrea cucullata) from Laft Port - Qeshm Island. Iran J Fish Sci 16(3): 897-906. http:// aquaticcommons.org/id/eprint/23127

American Meteorological Society (2012) Freshwater, Glossary of Meteorology. Available online http://glossary.ametsoc.org/wiki/ Freshwater. Accessed 18 Aug 2020
Anderson PJ, Warrack S, Langen V, Challis JK, Hanson ML, Rennie MD (2017) Microplastic contamination in lake Winnipeg, Canada. Environ Pollut 225:223-231. https://doi.org/10.1016/j. envpol.2017.02.072

Andrady AL (2011) Microplastics in the marine environment. Mar Pollut Bul 62(8):1596-1605. https://doi.org/10.1016/j.marpol bul.2011.05.030

Bagheri T, Gholizadeh M, Abarghouei S et al (2020) Microplastics distribution, abundance and composition in sediment, fishes and benthic organisms of the Gorgan Bay. Caspian sea Chemosphere 257:127201. https://doi.org/10.1016/j.chemosphere.2020.127201

Baldwin AK, Corsi SR, Mason SA (2016) Plastic debris in 29 Great Lakes tributaries: relations to watershed attributes and hydrology. Environ Sci Technol 50(19):10377-10385. https://doi.org/ 10.1021/acs.est.6b02917

Ballent A, Corcoran PL, Madden O, Helm PA, Longstaffe FJ (2016) Sources and sinks of microplastics in Canadian Lake Ontario nearshore, tributary and beach sediments. Mar Pollut Bull 110(1):383-395. https://doi.org/10.1016/j.marpolbul.2016.06. 037

Barrows AP, Christiansen KS, Bode ET, Hoellein TJ (2018) A watershed-scale, citizen science approach to quantifying microplastic concentration in a mixed land-use river. Water Res 147:382-392. https://doi.org/10.1016/j.watres.2018.10.013

Barrows AP, Neumann CA, Berger ML, Shaw SD (2017) Grab vs. neuston tow net: a microplastic sampling performance comparison and possible advances in the field. Anal Methods 9(9):1446-1453

Blair RM, Waldron S, Phoenix VR, Gauchotte-Lindsay C (2019) Microscopy and elemental analysis characterisation of microplastics in sediment of a freshwater urban river in Scotland. UK Environ Sci Pollut Res 26(12):12491-12504. https://doi.org/ 10.1007/s11356-019-04678-1

Blašković A, Guerranti C, Fastelli P, Anselmi S, Renzi M (2018) Plastic levels in sediments closed to Cecina river estuary (Tuscany, Italy). Mar Pollut Bull 135:105-109. https://doi.org/ 10.1016/j.marpolbul.2018.07.021

Blettler MC, Ulla MA, Rabuffetti AP, Garello N (2017) Plastic pollution in freshwater ecosystems: macro-, meso-, and microplastic debris in a floodplain lake. Environ Monit Assess 189(11):1-13. https://doi.org/10.1007/s10661-017-6305-8

Bordós G, Urbányi B, Micsinai A et al (2019) Identification of microplastics in fish ponds and natural freshwater environments of the Carpathian basin, Europe. Chemosphere 216:110-116. https://doi.org/10.1016/j.chemosphere.2018.10.110

Brandsma SH, Nijssen P, Van Velzen MJM., Leslie HA (2013) Microplastics in river suspended particulate matter and sewage treatment plants. Report R14/02, Institute for environmental studies. University Amsterdam, Netherland. Available online https://puc.overheid.nl/rijkswaterstaat/doc/PUC_147662_31/. Accessed 14 Feb 2020

Braun U, Jekel M, Gerdts G, Ivleva N, Reiber J (2018) Microplastics Analytics: Sampling, Preparation and Detection Methods. Discussion Paper within the scope of the research focus. Plastics in the Environment Sources Sinks Solutions. Discussion paper. German Federal Ministry of Education and Research, Germany. Available online https://bmbf-plastik.de/sites/default/files/201812/Discussion\%20Paper\%20Mikroplastics\%20Analytics\% 20Nov\%202018.pdf. Accessed 26 November 2020

Brennecke D, Duarte B, Paiva F, Caçador I, Canning-Clode J (2016) Microplastics as vector for heavy metal contamination from the marine environment. Estuarine Coastal Shelf Sci 178:189-195. https://doi.org/10.1016/j.ecss.2015.12.003

Briggs E, de Moura EAB, Furusawa HA, Cotrim MEB, Oguzie EE, Lugao AB (2019) Microplastics: a novel method for surface wate sampling ad sample extraction in Elechi Creek, Rivers State, 
Nigeria. In: Li B et al (eds) Characterization of minerals, metals, and materials the minerals, metals and materials 2019 series. Springer, Cham

Browne MA, Galloway TS, Thompson RC (2010) Spatial patterns of plastic debris along estuarine shorelines. Environ Sci Technol 44(9):3404-3409. https://doi.org/10.1021/es903784e

Campanale C, Stock F, Massarelli C et al (2020) Microplastics and their possible sources: the example of Ofanto river in Southeast Italy. Environ Pollut 258:113284. https://doi.org/10.1016/j. envpol.2019.113284

Campbell SH, Williamson PR, Hall BD (2017) Microplastics in the gastrointestinal tracts of fish and the water from an urban prairie creek. Facets 2(1):395-409. https://doi.org/10.1139/facets-20170008

Carpenter EJ, Anderson SJ, Harvey GR, Miklas HP, Peck BB (1972) Polystyrene spherules in coastal waters. Science 178(4062):749-750. https://doi.org/10.1126/science.178.4062. 749

Carpenter EJ, Smith KL (1972) Plastics on the Sargasso Sea surface. Sci 175(4027):1240-1241. https://doi.org/10.1126/science.175. 4027.1240

Castañeda RA, Avlijas S, Simard MA, Ricciardi A (2014) Microplastic pollution in St. Lawrence river sediments. Can J Fish Aquat Science 71(12): 1767-1771

Cedro A, Cleary J (2015) Microplastics in Irish freshwaters: a preliminary study. In Lekkas TD (ed) Proceedings of the 14th International Conference on Environmental Science and Technology, Rhodes, Greece 3:1666-1669. Available online https:// www.researchgate.net/profile/John-Cleary-9/publication/ 281619904_Microplastics_in_Irish_freshwaters_A_preliminary_ study/links/57750b1e08aead7ba06fbfef/Microplastics-in-Irishfreshwaters-A-preliminary-study.pdf. Accessed 13 Jan 2020.

Colton JB, Knapp FD, Burns BR (1974) Plastic particles in surface waters of the northwestern Atlantic. Sci 185(4150):491-497. http://www.jstor.org/stable/1738284

Connors KA, Dyer SD, Belanger SE (2017) Advancing the quality of environmental microplastic research. Environ Toxicol Chem 36(7):1697-1703. https://doi.org/10.1002/etc.3829

Corcoran PL, Belontz SL, Ryan K, Walzak MJ (2019) Factors controlling the distribution of microplastic particles in Benthic sediment of the Thames River. Canada Environ Sci Technol 54(2):818-825. https://doi.org/10.1021/acs.est.9b04896

Corcoran PL, Norris T, Ceccanese T et al (2015) Hidden plastics of Lake Ontario, Canada and their potential preservation in the sediment record. Environ Pollut 204:17-25. https://doi.org/10. 1016/j.envpol.2015.04.009

Costa CQ, Cruz J, Martins J, Teodósio MAA, Jockusch S, Ramamurthy V, Da Silva JP (2021) Fluorescence sensing of microplastics on surfaces. Environ Chem Lett. https://doi.org/10. 1007/s10311-020-01136-0

Cox K (2018) Distribution, Abundance, and Spatial Variability of Microplastic Pollution in Surface Waters of Lake Superior. Dissertation, University of Waterloo

Crawford C, Quinn B (2017) Microplastic collection techniques. In: Crawford B, Quinn B (eds) Microplastic pollutants. Elsevier Inc, Amsterdam

de Sá LC, Oliveira M, Ribeiro F, Rocha TL, Futter MN (2018) Studies of the effects of microplastics on aquatic organisms: what do we know and where should we focus our efforts in the future? Sci Total Environ 645:1029-1039. https://doi.org/10. 1016/j.scitotenv.2018.07.207

Dean BY, Corcoran PL, Helm PA (2018) Factors influencing microplastic abundances in nearshore, tributary and beach sediments along the Ontario shoreline of Lake Erie. J Great Lakes Res 44(5):1002-1009. https://doi.org/10.1016/j.jglr.2018. 07.014
Deocaris CC, Allosada JO, Ardiente LT et al (2019) Occurrence of microplastic fragments in the Pasig River. H2Open J 2(1):92-100. https://doi.org/10.2166/h2oj.2019.001

Di M, Liu X, Wang W, Wang J (2019) Manuscript prepared for submission to environmental toxicology and pharmacology pollution in drinking water source areas: Microplastics in the Danjiangkou Reservoir, China. Environ Toxicol Pharmacol 65:82-89. https://doi.org/10.1016/j.etap.2018.12.009

Di M, Wang J (2018) Microplastics in surface waters and sediments of the Three Gorges Reservoir, China. Sci Total Environ 616:1620-1627. https://doi.org/10.1016/j.scitotenv.2017.10.150

Dikareva N, Simon KS (2019) Microplastic pollution in streams spanning an urbanisation gradient. Environ Pollut 250:292-299. https://doi.org/10.1016/j.envpol.2019.03.105

Ding J, Zhang S, Razanajatovo RM, Zou H, Zhu W (2018) Accumulation, tissue distribution, and biochemical effects of polystyrene microplastics in the freshwater fish red tilapia (Oreochromis niloticus). Environ Pollut 238:1-9. https://doi.org/ 10.1016/j.envpol.2018.03.001

Ding L et al (2019) Microplastics in surface waters and sediments of the Wei River, in the northwest of China. Sci Total Environ 667:427-434. https://doi.org/10.1016/j.scitotenv.2019.02.332

Dong M, Luo Z, Jiang Q, Xing X, Zhang Q, Sun Y (2020) The rapid increases in microplastics in urban lake sediments. Sci Rep 10(1):1-10. https://doi.org/10.1038/s41598-020-57933-8

Dris R, Gasperi J, Rocher V, Saad M, Renault N, Tassin B (2015) Microplastic contamination in an urban area: a case study in Greater Paris. Environ Chem 12(5):592-599. https://doi.org/10. 1071/EN14167

Dris R, Gasperi J, Rocher V, Tassin B (2018) Synthetic and nonsynthetic anthropogenic fibers in a river under the impact of Paris Megacity: Sampling methodological aspects and flux estimations. Sci Total Environ 618:157-164. https://doi.org/10. 1016/j.scitotenv.2017.11.009

Dubaish F, Liebezeit G (2013) Suspended microplastics and black carbon particles in the Jade system, southern North Sea. Water Air Soil Pollut 224(2):1352. https://doi.org/10.1007/s11270-012$1352-9$

Egessa R, Nankabirwa A, Basooma R, Nabwire R (2020) Occurrence, distribution and size relationships of plastic debris along shores and sediment of northern Lake Victoria. Environ Pollut 257:113442. https://doi.org/10.1016/j.envpol.2019.113442

Enders K, Käppler A, Biniasch O et al (2019) Tracing microplastics in aquatic environments based on sediment analogies. Sci Rep 9(1):1-15. https://doi.org/10.1038/s41598-019-50508-2

Eo S, Hong SH, Song YK, Han GM, Shim WJ (2019) Spatiotemporal distribution and annual load of microplastics in the Nakdong River, South Korea. Water Res 160:228-237. https://doi.org/10. 1016/j.watres.2019.05.053

Eriksen M, Mason S, Wilson S, Box C, Zellers A, Edwards W, Amato $S$ (2013) Microplastic pollution in the surface waters of the Laurentian Great Lakes. Mar Pollut Bull 77(1-2):177-182. https://doi.org/10.1016/j.marpolbul.2013.10.007

Estahbanati S, Fahrenfeld NL (2016) Influence of wastewater treatment plant discharges on microplastic concentrations in surface water. Chemosphere 162:277-284. https://doi.org/10. 1016/j.chemosphere.2016.07.083

Fan Y, Zheng K, Zhu Z, Chen G, Peng X (2019) Distribution, sedimentary record, and persistence of microplastics in the Pearl River catchment, China. Environ Pollut 251:862-870. https:// doi.org/10.1016/j.envpol.2019.05.056

Farrell P, Nelson K (2013) Trophic level transfer of microplastic: Mytilus edulis (L.) to Carcinus maenas (L.). Environ Pollut 177:1-3. https://doi.org/10.1016/j.envpol.2013.01.046 
Faure F, Corbaz M, Baecher H, de Alencastro L (2012) Pollution due to plastics and microplastics in Lake Geneva and in the Mediterranean Sea. Arch des Sci 65:157-164

Faure F, Demars C, Wieser O, Kunz M, De Alencastro LF (2015) Plastic pollution in Swiss surface waters: nature and concentrations, interaction with pollutants. Environ Chem 12(5):582-591. https://doi.org/10.1071/EN14218

Firdaus M, Trihadiningrum Y, Lestari P (2020) Microplastic pollution in the sediment of Jagir Estuary, Surabaya City. Indonesia Mar Pollut Bull 150:110790. https://doi.org/10.1016/j.marpolbul. 2019.110790

Fischer EK, Paglialonga L, Czech E, Tamminga M (2016) Microplastic pollution in lakes and lake shoreline sediments-a case study on Lake Bolsena and Lake Chiusi (central Italy). Environ Pollut 213:648-657. https://doi.org/10.1016/j.envpol.2016.03.012

Free CM, Jensen OP, Mason SA, Eriksen M, Williamson NJ, Boldgiv B (2014) High-levels of microplastic pollution in a large, remote, mountain lake. Mar Pollut Bull 85(1):156-163. https://doi.org/ 10.1016/j.marpolbul.2014.06.001

Gallagher A, Rees A, Rowe R, Stevens J, Wright P (2016) Microplastics in the Solent estuarine complex, UK: an initial assessment. Mar Pollut Bull 102(2):243-249. https://doi.org/10. 1016/j.marpolbul.2015.04.002

Geyer R, Jambeck JR, Law KL (2017) Production, use, and fate of all plastics ever made. Sci Adv 3(7):e1700782. https://doi.org/10. 1126/sciadv. 1700782

Gorrasi G, Sorrentino A, Lichtfouse E (2020) Back to plastic pollution in COVID times. Environ Chem Lett 19(1):1-4. https:// doi.org/10.1007/s10311-020-01129-z

Gray AD, Wertz H, Leads RR, Weinstein JE (2018) Microplastic in two South Carolina Estuaries: occurrence, distribution, and composition. Mar Pollut Bull 128:223-233. https://doi.org/10. 1016/j.marpolbul.2018.01.030

Grbić J, Helm P, Athey S, Rochman CM (2020) Microplastics entering northwestern Lake Ontario are diverse and linked to urban sources. Water Res 174:115623. https://doi.org/10.1016/j. watres.2020.115623

Green DS (2016) Effects of microplastics on European flat oysters, Ostrea edulis and their associated benthic communities. Environ Pollut 216:95-103. https://doi.org/10.1016/j.envpol.2016.05.043

Hamidian AH, Jafari Ozumchelouei E, Feizi F, Wu C, Zhang Y, Yang M (2021) A review on the characteristics of microplastics in wastewater treatment plants: a source for toxic chemicals. J Clean Prod 295:126480. https://doi.org/10.1016/j.jclepro.2021. 126480

Hamidian AH, Razeghi N, Zhang Y, Yang M (2019) Spatial distribution of arsenic in groundwater of Iran, a review. J Geochem Explor 201:88-98. https://doi.org/10.1016/j.gexplo. 2019.03.014

Hamidian AH, Zareh Reshqueih M, Poorbagher H, Ashrafi VL, S, (2016) Heavy metal bioaccumulation in sediment, common reed, algae and blood worm from the Shoor River. Iran J Toxicol Environ 32(3):398-409. https://doi.org/10.1177/ 0748233713500835

Han M, Niu X, Tang M, Zhang BT, Wang G, Yue W, Zhu J (2020) Distribution of microplastics in surface water of the lower Yellow River near estuary. Sci Total Environ 707:135601. https://doi.org/10.1016/j.scitotenv.2019.135601

Hartmann NB, Rist S, Bodin J, Jensen LH, Schmidt SN, Mayer P, Baun A (2017) Microplastics as vectors for environmental contaminants: exploring sorption, desorption, and transfer to biota. Integr Environ Assess Manage 13(3):488-493. https://doi. org/10.1002/ieam.1904

He B, Goonetilleke A, Ayoko GA, Rintoul L (2020) Abundance, distribution patterns, and identification of microplastics in
Brisbane river sediments. Australia Sci Total Environ 700:134467. https://doi.org/10.1016/j.scitotenv.2019.134467

Hendrickson E, Minor EC, Schreiner K (2018) Microplastic abundance and composition in western Lake superior as determined via microscopy, Pyr-GC/MS, and FTIR. Environ Sci Technol 52(4):1787-1796. https://doi.org/10.1021/acs.est.7b05829

Hidalgo-Ruz V, Gutow L, Thompson RC, Thiel M (2012) Microplastics in the marine environment: a review of the methods used for identification and quantification. Environ Sci Technol 46(6):3060-3075. https://doi.org/10.1021/es2031505

Hitchcock JN, Mitrovic SM (2019) Microplastic pollution in estuaries across a gradient of human impact. Environ Pollut 247:457-466. https://doi.org/10.1016/j.envpol.2019.01.069

Holland ER, Mallory ML, Shutler D (2016) Plastics and other anthropogenic debris in freshwater birds from Canada. Sci Total Environ 571:251-258. https://doi.org/10.1016/j.scitotenv.2016. 07.158

Horton AA, Walton A, Spurgeon DJ, Lahive E, Svendsen C (2017) Microplastics in freshwater and terrestrial environments: Evaluating the current understanding to identify the knowledge gaps and future research priorities. Sci Total Environ 586:127-141. https://doi.org/10.1016/j.scitotenv.2017.01.190

Hu L, Chernick M, Hinton DE, Shi H (2018) Microplastics in small waterbodies and tadpoles from Yangtze River Delta. China Environ Sci Technol 52(15):8885-8893. https://doi.org/10.1021/ acs.est.8b02279

Huang Y, Tian M, Jin F, Chen M, Liu Z, Mu HS (2020) Coupled effects of urbanization level and dam on microplastics in surface waters in a coastal watershed of Southeast China. Mar Pollut Bul 154:111089. https://doi.org/10.1016/j.marpolbul.2020.111089

Huppertsberg S, Knepper TP (2018) Instrumental analysis of microplastics-benefits and challenges. Anal Bioanal Chem 410(25):6343-6352. https://doi.org/10.1007/s00216-018-1210-8

Hurley R, Woodward J, Rothwell JJ (2018) Microplastic contamination of river beds significantly reduced by catchment-wide flooding. Nat Geosci 11(4):251-257. https://doi.org/10.1038/ s41561-018-0080-1

Imhof HK, Ivleva NP, Schmid J, Niessner R, Laforsch C (2013) Contamination of beach sediments of a subalpine lake with microplastic particles. Curr Biol 23(19):R867-R868. https://doi. org/10.1016/j.cub.2013.09.001

Imhof HK, Laforsch C, Wiesheu AC et al (2016) Pigments and plastic in limnetic ecosystems: a qualitative and quantitative study on microparticles of different size classes. Water Res 98:64-74. https://doi.org/10.1016/j.watres.2016.03.015

Imhof HK, Wiesheu AC, Anger PM, Niessner R, Ivleva NP, Laforsch $\mathrm{C}$ (2018) Variation in plastic abundance at different lake beach zones-a case study. Sci Total Environ 613:30-537. https://doi. org/10.1016/j.scitotenv.2017.08.300

Irfan M, Qadir A, Mumtaz M, Ahmad SR (2020) An unintended challenge of microplastic pollution in the urban surface water system of Lahore. Pakistan Environ Sci Pollut Res 27(14):16718-16730. https://doi.org/10.1007/s11356-02008114-7

Jafari Ozumchelouei E, Hamidian AH, Zhang Y, Yang M (2020) Physicochemical properties of antibiotics: a review with an emphasis on detection in the aquatic environment. Water Environ Res 92:177-188. https://doi.org/10.1002/wer.1237

Wang JD, Peng J, Tan Z, Gao Y, Zhan Z, Chen Q, Cai L (2017a) Microplastics in the surface sediments from the Beijiang River littoral zone: composition, abundance, surface textures and interaction with heavy metals. Chemosphere 171:248-258. https://doi.org/10.1016/j.chemosphere.2016.12.074

Jiang C, Yin L, Li Z, Wen X, Luo X, Hu S, Liu Y (2019) Microplastic pollution in the rivers of the Tibet Plateau. Environ Pollut 249:91-98. https://doi.org/10.1016/j.envpol.2019.03.022 
Jiang C, Yin L, Wen X, Du C, Wu L, Long Y, Pan H (2018) Microplastics in sediment and surface water of west dongting lake and south dongting lake: abundance, source and composition. Int J Environ Res Public Health 15(10):2164. https://doi. org/10.3390/ijerph15102164

Kapp KJ, Yeatman E (2018) Microplastic hotspots in the Snake and Lower Columbia rivers: a journey from the greater yellowstone ecosystem to the Pacific ocean. Environ Pollut 241:1082-1090. https://doi.org/10.1016/j.envpol.2018.06.033

Karlsson TM, Kärrman A, Rotander A, Hassellöv M (2020) Comparison between manta trawl and in situ pump filtration methods, and guidance for visual identification of microplastics in surface waters. Environ Sci Pollut Res 27(5):5559-5571. https://doi.org/10.1007/s11356-019-07274-5

Kataoka T, Nihei Y, Kudou K, Hinata H (2019) Assessment of the sources and inflow processes of microplastics in the river environments of Japan. Environ Pollut 244:958-965. https://doi. org/10.1016/j.envpol.2018.10.111

Kershaw P, Katsuhiko S, Lee S, Samseth J, Woodring D (2011) Plastic debris in the ocean. In: UNEP Year Book 2011: Emerging Issues in our Global Environment, Nairobi, Kenya, pp 21

Klein S, Worch E, Knepper TP (2015) Occurrence and spatial distribution of microplastics in river shore sediments of the Rhine-Main area in Germany. Environ Sci Technol 49(10):6070-6076. https://doi.org/10.1021/acs.est.5b00492

Koelmans AA, Bakir A, Burton GA, Janssen CR (2016) Microplastic as a vector for chemicals in the aquatic environment: critical review and model-supported reinterpretation of empirical studies. Environ Sci Technol 50(7):3315-3326. https://doi.org/10. 1021/acs.est.5b06069

Lahens L, Strady E, Kieu-Le TC, Drisn R, Boukerma K, Rinnert E, Tassin B (2018) Macroplastic and microplastic contamination assessment of a tropical river (Saigon River, Vietnam) transversed by a developing megacity. Environ Pollut 236:661-671. https://doi.org/10.1016/j.envpol.2018.02.005

Lasee S, Mauricio J, Thompson WA et al (2017) Microplastics in a freshwater environment receiving treated wastewater effluent. Integr Environ Assess Manage 13(3):528-532. https://doi.org/ 10.1002/ieam. 1915

Lechner A, Keckeis H, Lumesberger-Loisl F et al (2014) The Danube so colourful: a potpourri of plastic litter outnumbers fish larvae in Europe's second largest river. Environ Pollut 188:177-181. https://doi.org/10.1016/j.envpol.2014.02.006

Leslie HA, Brandsma SH, Van Velzen MJM, Vethaak AD (2017) Microplastics en route: field measurements in the Dutch river delta and Amsterdam canals, wastewater treatment plants, North Sea sediments and biota. Environ Int 101:133-142. https://doi. org/10.1016/j.envint.2017.01.018

Li L, Geng S, Wu C, Song K, Sun F, Visvanathan C, Wang Q (2019) Microplastics contamination in different trophic state lakes along the middle and lower reaches of Yangtze River Basin. Environ Pollut 254:112951. https://doi.org/10.1016/j.envpol.2019.07.119

Liedermann M, Gmeiner P, Pessenlehner S, Haimann M, Hohenblum P, Habersack H (2018) A methodology for measuring microplastic transport in large or medium rivers. Water 10(4):414. https:// doi.org/10.3390/w10040414

Lin L, Zuo LZ, Peng JP, Cai LQ et al (2018) Occurrence and distribution of microplastics in an urban river: a case study in the Pearl River along Guangzhou City, China. Sci Total Environ 644:375-381. https://doi.org/10.1016/j.scitotenv.2018.06.327

Liu F, Olesen KB, Borregaard AR, Vollertsen J (2019) Microplastics in urban and highway stormwater retention ponds. Sci Total Environ 671:992-1000. https://doi.org/10.1016/j.scitotenv.2019. 03.416
Luo W, Su L, Craig NJ, Du F, Wu C, Shi H (2019) Comparison of microplastic pollution in different water bodies from urban creeks to coastal waters. Environ Pollut 246:174-182. https:// doi.org/10.1016/j.envpol.2018.11.081

Lusher A, Buenaventura NT, Eidsvoll D, Thrane JE, Økelsrud A, Jartun M (2018) Freshwater microplastics in Norway: A first look at sediment, biota and historical plankton samples from Lake Mjøsa and Lake Femunden. Norwegian Insititute for Water Research. Available online http://hdl.handle.net/11250/2588713. Accessed 20 Apr 2020

Lusher AL, Welden NA, Sobral P, Cole M (2017) Sampling, isolating and identifying microplastics ingested by fish and invertebrates. Anal Methods 9(9):1346-1360. https://doi.org/10.1039/ C6AY02415G

Mani T, Blarer P, Storck FR, Pittroff M, Wernicke T, BurkhardtHolm P (2019a) Repeated detection of polystyrene microbeads in the lower Rhine River. Environ Pollut 245:634-641. https:// doi.org/10.1016/j.envpol.2018.11.036

Mani T, Burkhardt-Holm P (2020) Seasonal microplastics variation in nival and pluvial stretches of the Rhine River-From the Swiss catchment towards the North Sea. Sci Total Environ 707:135579. https://doi.org/10.1016/j.scitotenv.2019.135579

Mani T, Hauk A, Walter U, Burkhardt-Holm P (2015) Microplastics profile along the Rhine River. Sci Rep 5(1):1-7. https://doi.org/ 10.1038/srep17988

Mani T, Primpke S, Lorenz C, Gerdts G, Burkhardt-Holm P (2019b) Microplastic pollution in benthic midstream sediments of the Rhine River. Environ Sci Technol 53(10):6053-6062. https://doi. org/10.1021/acs.est.9b01363

Mansouri B, Pourkhabbaz A, Ebrahimpour M, Hamidian BHAH (2013) Bioaccumulation and elimination rate of cobalt in Capoeta fusca under controlled conditions. Chem Speciat Bioavailab 25(1):52-56. https://doi.org/10.3184/ $095422913 X 13581898658634$

Mao Y, Li H, Gu W, Yang G, Liu Y, He Q (2020a) Distribution and characteristics of microplastics in the Yulin River, China: Role of environmental and spatial factors. Environ Pollut 265:115033. https://doi.org/10.1016/j.envpol.2020.115033

Mao R, Hu Y, Zhang S, Wu R, Guo X (2020b) Microplastics in the surface water of Wuliangsuhai Lake, northern China. Sci Total Environ. https://doi.org/10.1016/j.scitotenv.2020.137820

Martínez Silva P, Nanny MA (2020) Impact of microplastic fibers from the degradation of nonwoven synthetic textiles to the Magdalena river water column and river sediments by the city of Neiva, Huila (Colombia). Water 12(4):1210. https://doi.org/10. 3390/w12041210

Mason SA, Kammin L, Eriksen M, Aleid G, Wilson S, Riley BCA (2016) Pelagic plastic pollution within the surface waters of Lake Michigan, USA. J Great Lakes Res 42(4):753-759. https:// doi.org/10.1016/j.jglr.2016.05.009

Mathalon A, Hill P (2014) Microplastic fibers in the intertidal ecosystem surrounding Halifax Harbor. Nova Scotia Mar Pollut Bull 81(1):69-79. https://doi.org/10.1016/j.marpolbul.2014.02. 018

McCormick A, Hoellein TJ, Mason SA, Schluep J, Kelly JJ (2014) Microplastic is an abundant and distinct microbial habitat in an urban river. Environ Sci Technol 48(20):11863-11871. https:// doi.org/10.1021/es503610r

McCormick AR, Hoellein TJ, London MG, Hittie J, Scott JW, Kelly JJ (2016) Microplastic in surface waters of urban rivers: concentration, sources, and associated bacterial assemblages. Ecosphere 7(11):e01556. https://doi.org/10.1002/ecs2.1556

McDevitt C, Perez L, Kapp K (2016) The presence of microplastic in freshwater systems: Snake river and Palisades Reservoir. Department of Health and Science, Central Wyoming 
University. Available https://mountainscholar.org/handle/20. 500.11919/2598/. Accessed 3 October 2019

McNeish RE, Kim LH, Barrett HA, Mason SA, Kelly JJ, Hoellein TJ (2018) Microplastic in riverine fish is connected to species traits. Sci Rep 8(1):1-12. https://doi.org/10.1038/s41598-018-29980-9

Merga LB, Redondo-Hasselerharm PE, Van den Brink PJ, Koelmans AA (2020) Distribution of microplastic and small macroplastic particles across four fish species and sediment in an African lake. Sci Total Environ 741:140527. https://doi.org/10.1016/j.scito tenv.2020.140527

Michida Y, Chavanich S, Chiba S, Cordova MR, Cozsar Cabanas A, Glagani F Kozlovskii N (2019) Guidelines for Harmonizing Ocean Surface Microplastic Monitoring Methods. Version 1.1. Ministry of the Environment, JAPAN. Available online https:// repository.oceanbestpractices.net/bitstream/handle/11329/1361/ Guildlines_ver.1.1_v28_200618.pdf?sequence=1\&isAllowed=y. Accessed Feb 2021

Miller RZ, Watts AJ, Winslow BO, Galloway TS, Barrows AP (2017) Mountains to the sea: river study of plastic and non-plastic microfiber pollution in the northeast USA. Mar Pollut Bull 124(1):245-251. https://doi.org/10.1016/j.marpolbul.2017.07. 028

Mintenig SM, Kooi M, Erich MW, Primpke S, Redondo-Hasselerharm PE, Dekker SC, van Wezel AP (2020) A systems approach to understand microplastic occurrence and variability in Dutch riverine surface waters. Water Res 176:115723. https://doi.org/ 10.1016/j.watres.2020.115723

Mirzajani A, Hamidian AH, Karami M (2016) Distribution and abundance of fish in the southwest of Caspian Sea coastal waters. Russ J Mar Bio 42(2):178-189. https://doi.org/10.1134/ S1063074016020073

Mirzajani A, Hamidian AH, Karami M (2017) Metal bioaccumulation in representative organisms from different trophic levels of the Caspian Sea. Iran J Fish Sci 15(3):1027-1043. http://aquatic commons.org/id/eprint/22930

Mirzajani A, Hamidian AH, Bagheri S, Karami M (2015) Possible effect of Balanus improvisus on Cerastoderma glaucum distribution in the south-western Caspian Sea. J Mar Biol Assoc U K 96(5):1031-1040. https://doi.org/10.1017/S0025315415000788

Mojoudi F, Hamidian AH, Goodarzian N, Eagderi S (2018) Effective removal of heavy metals from aqueous solution by porous activated carbon/thiol functionalized graphene oxide composite. Desalin Water Treat 124:106-116. https://doi.org/10.5004/dwt. 2018.22695

Mojoudi F, Hamidian AH, Zhang Y, Yang M (2019) Synthesis and evaluation of Activated Carbon/Nanoclay/ Thiolated Graphene Oxide Nanocomposite for Lead (II) Removal from Aqueous Solution. Water Sci Technol 79(3):466-479. https://doi.org/10. 2166/wst.2019.071

Moore CJ, Lattin GL, Zellers AF (2011) Quantity and type of plastic debris flowing from two urban rivers to coastal waters and beaches of Southern California. J Coas Zone Manag 11(1):65-73. https://www.redalyc.org/pdf/3883/388340132008. pdf

Naidoo T, Glassom D, Smit AJ (2015) Plastic pollution in five urban estuaries of KwaZulu-Natal. South Africa Mar Pollut Bull 101(1):473-480. https://doi.org/10.1016/j.marpolbul.2015.09. 044

Nan B, Su L, Kellar C, Craig NJ, Keough MJ, Pettigrove V (2020) Identification of microplastics in surface water and Australian freshwater shrimp Paratya australiensis in Victoria. Australia Environ Pollut 259:113865. https://doi.org/10.1016/j.envpol. 2019.113865

Naqash N, Prakash S, Kapoor D, Singh R (2020) Interaction of freshwater microplastics with biota and heavy metals: a review.
Environ Chem Lett 18(6):1813-1824. https://doi.org/10.1007/ s10311-020-01044-3

Neto JAB, Gaylarde C, Beech I, Bastos AC, da Silva QV, de Carvalho DG (2019) Microplastics and attached microorganisms in sediments of the Vitória bay estuarine system in SE Brazil. Ocean Coast Manage 169:247-253. https://doi.org/10.1016/j. ocecoaman.2018.12.030

Noik VJ, Tuah PM (2015) A first survey on the abundance of plastics fragments and particles on two sandy beaches in Kuching, Sarawak. Malaysia Conf Ser Mater Sci Eng 78(1):012035. https://doi.org/10.1088/1757-899X/78/1/012035

Ogonowski M, Schür C, Gorokhova JA (2016) The effects of natural and anthropogenic microparticles on individual fitness in Daphnia magna. PLoS ONE 11(5):e0155063. https://doi.org/10.1371/ journal.pone.0155063

Olesen KB, Stephansen DA, van Alst N, Vollertsen J (2019) Microplastics in a stormwater pond. Water 11(7):1466. https:// doi.org/10.3390/w11071466

Othman AR, Hasan HA, Muhamad MH et al (2021) Microbial degradation of microplastics by enzymatic processes: a review. Environ Chem Lett. https://doi.org/10.1007/s10311-021-01197-9

Padash Barmchi Z, Hamidian AH, Khorasani N, Kazemzad M, McCabe A, Halog A (2015) Environmental life cycle assessments of emerging anode materials for Li-Ion batteries-metal oxide NPs. Environ Prog Sustain Energy 34(6):1740-1747. https://doi.org/10.1002/ep.12148

Padervand M, Lichtfouse E, Robert D, Wang C (2020) Removal of microplastics from the environment. A Rev Environ Chem Lett 18(3):807-828. https://doi.org/10.1007/s10311-020-00983-1

Pariatamby A, Hamid FS, Bhatti MS, Anuar N, Anuar N (2020) Status of microplastic pollution in aquatic ecosystem with a case study on Cherating River, Malaysia. J Eng Technol Sci. https:// doi.org/10.5614/j.eng.technol.sci.2020.52.2.7

Pellini G, Gomiero A, Fortibuoni T, Ferrà C, Grati F, Tassetti AN, Scarcella G (2018) Characterization of microplastic litter in the gastrointestinal tract of Solea solea from the Adriatic Sea. Environ Pollut 234:943-952. https://doi.org/10.1016/j.envpol. 2017.12.038

Peng G, Xu P, Zhu B, Bai M, Li D (2018) Microplastics in freshwater river sediments in Shanghai, China: a case study of risk assessment in mega-cities. Environ Pollut 234:448-456. https:// doi.org/10.1016/j.envpol.2017.11.034

Peng G, Zhu B, Yang D, Su L, Shi H, Li D (2017) Microplastics in sediments of the Changjiang Estuary, China. Environ Pollut 225:283-290. https://doi.org/10.1016/j.envpol.2016.12.064

Plastics Europe (2018). Plastics-The facts 2018. An analysis of European plastics production, demand and waste data. Available online https://www.plasticseurope.org/application/files/6315/ 4510/9658/Plastics_the_facts_2018_AF_web.pdf. Accessed 18 Aug 2020

Rao Z, Niu S, Zhan N, Wang X, Song X (2020) Microplastics in sediments of River Yongfeng from Maanshan city, Anhui Province. China Bull Environ Contam Toxicol 104(2):166-172. https://doi.org/10.1007/s00128-019-02771-2

Razeghi N, Hamidian AH, Wu C, Zhang Y, Yang M (2020) Scientific studies on microplastics pollution in Iran: an in-depth review of the published articles. Mar Pollut Bull 162:111901. https://doi. org/10.1016/j.marpolbul.2020.111901

Reynolds C, Ryan PG (2018) Micro-plastic ingestion by waterbirds from contaminated wetlands in South Africa. Mar Pollut Bul 126:330-333. https://doi.org/10.1016/j.marpolbul.2017.11.021

Rezaei Kalvani S, Sharaai AH, Abd Manaf L, Hamidian AH (2019) Assessing ground and surface water scarcity indices using ground and surface water footprints in the Tehran province of Iran. Appl Ecol Env Res 17(2):4985-4997. https://doi.org/10. 15666/aeer/1702_49854997 
Rezania S, Park J, Din MFM, Taib SM, Talaiekhozani A, Yadav KK, Kamyab H (2018) Microplastics pollution in different aquatic environments and biota: a review of recent studies. Mar Pollut Bull 133:191-208.

Rodrigues MO, Abrantes N, Gonçalves FJM, Nogueira H, Marques JC, Gonçalves AMM (2018) Spatial and temporal distribution of microplastics in water and sediments of a freshwater system (Antuã River, Portugal). Sci Total Environ 633:1549-1559. https://doi.org/10.1016/j.scitotenv.2018.03.233

Rodrigues SM, Almeida CMR, Silva D, Cunha J, Antunes C, Freitas V, Ramos S (2019) Microplastic contamination in an urban estuary: abundance and distribution of microplastics and fish larvae in the Douro estuary. Sci Total Environ 659:1071-1081. https://doi.org/10.1016/j.scitotenv.2018.12.273

Sadri SS, Thompson RC (2014) On the quantity and composition of floating plastic debris entering and leaving the Tamar Estuary. Southwest England Mar Pollut Bull 81(1):55-60. https://doi.org/ 10.1016/j.marpolbul.2014.02.020

Sarijan S, Azman S, Said MIM, Andu Y, Zon NF (2018) Microplastics in sediment from Skudai and Tebrau river, Malaysia: a preliminary study. MATEC Web of Conf 250:06012. https://doi. org/10.1051/matecconf/201825006012

Setälä O, Fleming-Lehtinen V, Lehtiniemi M (2014) Ingestion and transfer of microplastics in the planktonic food web. Environ Pollut 185:77-83. https://doi.org/10.1016/j.envpol.2013.10.013

Sharma S, Chatterjee S (2017) Microplastic pollution, a threat to marine ecosystem and human health: a short review. Environ Sci Pollut Res 24(27):21530-21547. https://doi.org/10.1007/s11356017-9910-8

Shruti VC, Jonathan MP, Rodriguez-Espinosa PF, RodríguezGonzález F (2019) Microplastics in freshwater sediments of atoyac river basin, puebla city, Mexico. Environ Sci Pollut Res 654:154-163. https://doi.org/10.1016/j.scitotenv.2018.11.054

Sighicelli M, Pietrelli L, Lecce F, Iannilli V, Falconieri M, Coscia L, Zampetti G (2018) Microplastic pollution in the surface waters of Italian Subalpine Lakes. Environ Pollut 236:645-651. https:// doi.org/10.1016/j.envpol.2018.02.008

Simon-Sánchez L, Grelaud M, Garcia-Orellana J, Ziveri P (2019) River Deltas as hotspots of microplastic accumulation: The case study of the Ebro River (NW Mediterranean). Sci Total Environ 687:1186-1196. https://doi.org/10.1016/j.scitotenv.2019.06.168

Smith JA, Hodge JL, Kurtz BH, Garver JI (2017) The distribution of microplastic pollution in the Mohawk River. Mohawk Watershed Symposium. Available online https://www.researchgate. net/profile/John-Garver/publication/314543747_Proceedings_ of_the_2017_Mohawk_Watershed_Symposium_Union_Col lege_Schenectady_NY_17_Mb/links/

58c339a8aca272e36dd0467c/Proceedings-of-the-2017-MohawkWatershed-Symposium-Union-College-Schenectady-NY-17Mb.pdf\#page $=78$. Accessed 20 May 2020

Sruthy S, Ramasamy EV (2017) Microplastic pollution in Vembanad Lake, Kerala, India: the first report of microplastics in lake and estuarine sediments in India. Environ Pollut 222:315-322. https://doi.org/10.1016/j.envpol.2016.12.038

Su L, Cai H, Kolandhasamy P, Wu C, Rochman CM, Shi H (2018) Using the Asian clam as an indicator of microplastic pollution in freshwater ecosystems. Environ Pollut 234:347-355. https://doi. org/10.1016/j.envpol.2017.11.075

Su L, Xue Y, Li L, Yang D, Kolandhasamy P, Li D, Shi H (2016) Microplastics in Taihu lake, China. Environ Pollut 216:711-719. https://doi.org/10.1016/j.envpol.2016.06.036

Ta AT, Babel S, Haarstrick A (2020) Microplastics contamination in a high population density area of the Chao Phraya River ,Bangkok. J Eng Technol. https://doi.org/10.5614/j.eng.technol. sci.2020.52.4.6
Tamminga M, Stoewer SC, Fischer EK (2019) On the representativeness of pump water samples versus manta sampling in microplastic analysis. Environ Pollut 254:112970. https://doi. org/10.1016/j.envpol.2019.112970

Tan X, Yu X, Cai L, Wang J, Peng J (2019) Microplastics and associated PAHs in surface water from the Feilaixia Reservoir in the Beijiang River, China. Chemosphere 221:834-840.

Thompson RC, Moore CJ, Vom Saal FS, Swan SH (2009) Plastics, the environment and human health: current consensus and future trends. Philos Trans R Soc 364(1526):2153-2166. https://doi. org/10.1098/rstb.2009.0053

Thompson RC, Olsen Y, Mitchell RP, Davis A, Rowland SJ, John AW, Russell AE (2004) Lost at sea: Where is all the plastic? Science 304(5672):838-838. https://doi.org/10.1126/science. 1094559

Tibbetts J, Krause S, Lynch I, Sambrook Smith GH (2018) Abundance, distribution, and drivers of microplastic contamination in urban river environments. Water 10(11):1597. https://doi. org/10.3390/w10111597

Tofa TS, Kunjali KL, Paul S, Dutta J (2019) Visible light photocatalytic degradation of microplastic residues with zinc oxide nanorods. Environ Chem Lett 17(3):1341-1346. https:// doi.org/10.1007/s10311-019-00859-z

Toumi H, Abidli S, Bejaoui M (2019) Microplastics in freshwater environment: the first evaluation in sediments from seven water streams surrounding the lagoon of Bizerte (Northern Tunisia). Environ Sci Pollut Res 26(14):14673-14682. https://doi.org/10. 1007/s11356-019-04695-0

Turner S, Horton AA, Rose NL, Hall C (2019) A temporal sediment record of microplastics in an urban lake, London. UK J Paleolimnol 61(4):449-462. https://doi.org/10.1007/s10933019-00071-7

van der Wal M, van der Meulen M, Tweehuijsen G, Peterlin M, Palatinus A, Kovac Viršek M (2015) SFRA0025: Identification and Assessment of Riverine Input of (Marine) Litter. Report for Michail Papadoyannakis, DG Environment, United Kingdom, pp 186. Available online https://portal.helcom.fi/meetings/PRES SURE\%203-2015-278/MeetingDocuments/5-8_att\%20Final\% 20Report $\% 20$ for $\% 20 \mathrm{EC} \% 20 \mathrm{DG} \% 20 \mathrm{ENV} \% 20$-Identification\% 20and $\% 20$ Assessment $\% 20$ of $\% 20$ Riverine $\% 20$ Input $\% 20 \mathrm{of} \%$ 20Marine\%20Litter.pdf. Accessed 20 Feb 2020

Vaughan R, Turner SD, Rose NL (2017) Microplastics in the sediments of a UK urban lake. Environ Pollut 229:10-18. https:// doi.org/10.1016/j.envpol.2017.05.057

Vermaire JC, Pomeroy C, Herczegh SM, Haggart O, Murphy M (2017) Microplastic abundance and distribution in the open water and sediment of the Ottawa River, Canada, and its tributaries. Facets 2(1):301-314. https://doi.org/10.1139/facets2016-0070

Vianello A, Boldrin A, Guerriero P, Moschino V, Rella R, Sturaro A, Da Ros L (2013) Microplastic particles in sediments of Lagoon of Venice, Italy: first observations on occurrence, spatial patterns and identification. Estuarine Coastal Shelf Sci 130:54-61. https://doi.org/10.1016/j.ecss.2013.03.022

Wang C, Xing R, Sun M, Ling W, Shi W, Cui S, An L (2020a) Microplastics profile in a typical urban river in Beijing. Sci Total Environ 743:140708. https://doi.org/10.1016/j.scitotenv.2020. 140708

Wang G, Lu J, Tong Y, Liu Z, Zhou H, Xiayihazi N (2020b) Occurrence and pollution characteristics of microplastics in surface water of the Manas River Basin. China Sci Total Environ 710:136099. https://doi.org/10.1016/j.scitotenv.2019.136099

Wang Z, Qin Y, Li W, Yang W, Meng Q, Yang J (2019) Microplastic contamination in freshwater: first observation in Lake Ulansuhai, Yellow River Basin. China Environ Chem Lett 17(4):1821-1830. https://doi.org/10.1007/s10311-019-00888-8 
Warrack S, Challis JK, Hanson ML, Rennie MD (2017) Microplastics flowing into Lake Winnipeg: densities, sources, flux, and fish exposures. Proc Manit Undergrad Sci Eng Res 3:5-15. https:// doi.org/10.5203/pmuser.201730578

Watkins L, McGrattan S, Sullivan PJ, Walter MT (2019a) The effect of dams on river transport of microplastic pollution. Sci Total Environ 664:834-840. https://doi.org/10.1016/j.scitotenv.2019. 02.028

Watkins L, Sullivan PJ, Walter MT (2019b) A case study investigating temporal factors that influence microplastic concentration in streams under different treatment regimes. Environ Sci Pollut Res 26(21):21797-21807. https://doi.org/10.1007/s11356-01904663-8

Wen X, Du C, Xu P, Zeng G, Huang D, Yin L, Tan S (2018) Microplastic pollution in surface sediments of urban water areas in Changsha, China: abundance, composition, surface textures. Mar Pollut Bull 136:414-423. https://doi.org/10.1016/j.marpol bul.2018.09.043

Wessel CC, Lockridge GR, Battiste D, Cebrian J (2016) Abundance and characteristics of microplastics in beach sediments: insights into microplastic accumulation in northern Gulf of Mexico estuaries. Mar Pollut Bull 109(1):178-183. https://doi.org/10. 1016/j.marpolbul.2016.06.002

Wang WF, Ndungu AW, Li Z, Wang J (2017b) Microplastics pollution in inland freshwaters of China: a case study in urban surface waters of Wuhan, China. Sci Total Environ 575:1369-1374. https://doi.org/10.1016/j.scitotenv.2016.09.213

Wang WF, Yuan W, Chen Y, Wang J (2018a) Microplastics in surface waters of dongting lake and hong lake. Sci Total Environ 633:539-545. https://doi.org/10.1016/j.scitotenv.2018.03.211

Wang ZF, Su B, Xu X, Di D, Huang H, Mei K, Shang X (2018b) Preferential accumulation of small $(<300 \mu \mathrm{m})$ microplastics in the sediments of a coastal plain river network in eastern China. Water Res 144:393-401. https://doi.org/10.1016/j.watres.2018. 07.050

Willis KA, Eriksen R, Wilcox C, Hardesty BD (2017) Microplastic distribution at different sediment depths in an urban estuary. Front Mar Sci 4:419. https://doi.org/10.3389/fmars.2017.00419

Wu C, Zhang K, Xiong X (2018) Microplastic pollution in inland waters focusing on Asia. In: Wagner $\mathrm{M}$, Lambert $\mathrm{S}$ (eds) Freshwater Microplastics: emerging environmental contaminants? Springer, Heidelberg, pp 85-99

Wu P, Tang Y, Dang M, Wang S, Jin H, Liu Y, Cai Z (2020) Spatialtemporal distribution of microplastics in surface water and sediments of Maozhou River within Guangdong-Hong KongMacao Greater Bay Area. Sci Total Environ 717:135187. https:// doi.org/10.1016/j.scitotenv.2019.135187

Xiong X, Wu C, Elser JJ, Mei Z, Hao Y (2019) Occurrence and fate of microplastic debris in middle and lower reaches of the Yangtze River-from inland to the sea. Sci Total Environ 659:66-73. https://doi.org/10.1016/j.scitotenv.2018.12.313

Xiong X, Zhang K, Chen X, Shi H, Luo Z, Wu C (2018) Sources and distribution of microplastics in China's largest inland lakeQinghai Lake. Environ Pollut 235:899-906. https://doi.org/10. 1016/j.envpol.2017.12.081

Xu P, Peng G, Su L, Gao Y, Gao L, Li D (2018) Microplastic risk assessment in surface waters: A case study in the Changjiang Estuary, China. Mar Pollut Bull 133:647-654. https://doi.org/10. 1016/j.marpolbul.2018.06.020

Xu Q, Xing R, Sun M, Gao Y, An L (2020) Microplastics in sediments from an interconnected river-estuary region. Sci Total Environ 729:139025. https://doi.org/10.1016/j.scitotenv.2020. 139025

Yan M, Nie H, Xu K, He Y, Hu Y, Huang Y (2019) Microplastic abundance, distribution and composition in the Pearl River along Guangzhou city and Pearl River estuary, China. Chemosphere
217:879-886. https://doi.org/10.1016/j.chemosphere.2018.11. 093

Yin L, Jiang C, Wen X, Du C, Zhong W, Feng Z, Ma Y (2019) Microplastic pollution in surface water of urban lakes in Changsha, China. Int J Environ Res Public Health 16(9):1650. https://doi.org/10.3390/ijerph16091650

Yin L, Wen X, Du C, Jiang J, Wu L, Long ZY (2020) Comparison of the abundance of microplastics between rural and urban areas: a case study from East Dongting Lake. Chemosphere 244:125486. https://doi.org/10.1016/j.chemosphere.2019.125486

Yonkos LT, Friedel EA, Perez-Reyes AC, Ghosal S, Arthur CD (2014) Microplastics in four estuarine rivers in the Chesapeake Bay, USA. Environ Sci Technol 48(24):14195-14202. https:// doi.org/10.1021/es5036317

Yu X, Peng J, Wang J, Wang K, Bao S (2016) Occurrence of microplastics in the beach sand of the Chinese inner sea: the Bohai Sea. Environ Pollut 214:722-730. https://doi.org/10.1016/ j.envpol.2016.04.080

Yuan W, Liu X, Wang W, Di M, Wang J (2019) Microplastic abundance, distribution and composition in water, sediments, and wild fish from Poyang Lake, China. Ecotoxicol Environ Saf 170:180-187. https://doi.org/10.1016/j.ecoenv.2018.11.126

Zbyszewski M, Corcoran PL (2011) Distribution and degradation of fresh water plastic particles along the beaches of Lake Huron. Can Water Air Soil Pollut 220(1-4):365-372. https://doi.org/10. 1007/s11270-011-0760-6

Zbyszewski M, Corcoran PL, Hockin A (2014) Comparison of the distribution and degradation of plastic debris along shorelines of the Great Lakes North America. J Great Lakes Res 40(2):288-299. https://doi.org/10.1016/j.jglr.2014.02.012

Zhang K, Chen X, Xiong X, Ruan Y, Zhou H, Wu C, Lam PK (2019) The hydro-fluctuation belt of the three Gorges reservoir: source or sink of microplastics in the water? Environ Pollu 248:279-285. https://doi.org/10.1016/j.envpol.2019.02.043

Zhang K, Gong W, Lv J, Xiong X, Wu C (2015) Accumulation of floating microplastics behind the three Gorges Dam. Environ Pollut 204:117-123. https://doi.org/10.1016/j.envpol.2015.04. 023

Zhang K, Shi H, Peng J, Wang Y, Xiong X, Wu C, Lam PK (2018) Microplastic pollution in China's inland water systems: A review of findings, methods, characteristics, effects, and management. Sci Total Environ 630:1641-1653. https://doi.org/10.1016/j. scitotenv.2018.02.300

Zhang K, Su J, Xiong X, Wu X, Wu C, Liu J (2016) Microplastic pollution of lakeshore sediments from remote lakes in Tibet plateau, China. Environ Pollut 219:450-455. https://doi.org/10. 1016/j.envpol.2016.05.048

Zhang K, Xiong X, Hu H, Wu C, Bi Y, Wu Y, Liu J (2017) Occurrence and characteristics of microplastic pollution in Xiangxi Bay of Three Gorges Reservoir. China Environ Sci Technol 51(7):3794-3801. https://doi.org/10.1021/acs.est. $7 b 00369$

Zhang X, Leng Y, Liu X, Huang K, Wang J (2020) Microplastics' pollution and risk assessment in an urban river: a case study in the Yongjiang River, Nanning City. South China Exposure Health 12(2):141-151. https://doi.org/10.1007/s12403-01800296-3

Zhao S, Wang T, Zhu L, Xu P, Wang X, Gao L, Li D (2019) Analysis of suspended microplastics in the Changjiang Estuary: Implications for riverine plastic load to the ocean. Water Res 161:560-569. https://doi.org/10.1016/j.watres.2019.06.019

Zhao S, Zhu L, Li D (2015) Microplastic in three urban estuaries, China. Environ Pollut 206:597-604. https://doi.org/10.1016/j. envpol.2015.08.027

Zhao S, Zhu L, Wang T, Li D (2014) Suspended microplastics in the surface water of the Yangtze Estuary System, China: first 
observations on occurrence, distribution. Mar Pollut Bull 86(1-2):562-568. https://doi.org/10.1016/j.marpolbul.2014.06. 032

Zhou G, Wang Q, Zhang J, Li Q, Wang Y, Wang M, Huang X (2020) Distribution and characteristics of microplastics in urban waters of seven cities in the Tuojiang River basin. China Environ Res 189:109893. https://doi.org/10.1016/j.envres.2020.109893

Publisher's Note Springer Nature remains neutral with regard to jurisdictional claims in published maps and institutional affiliations. 